\title{
Global dynamical evolution of the ISM in star forming galaxies
}

\section{High resolution 3D simulations: Effect of the magnetic field}

\author{
M. A. de Avillez ${ }^{1}$ and D. Breitschwerdt ${ }^{2}$ \\ 1 Department of Mathematics, University of Évora, R. Romão Ramalho 59, 7000 Évora, Portugal \\ e-mail: mavillez@galaxy.lca.uevora.pt \\ 2 Institut für Astronomie, Universität Wien, Türkenschanzstr. 17, 1180 Wien, Austria \\ e-mail: breitschwerdt@astro.univie.ac.at
}

Received 8 October 2004 / Accepted 31 January 2005

\begin{abstract}
In star forming disk galaxies, matter circulation between stars and the interstellar gas, and, in particular the energy input by random and clustered supernova explosions, determine the dynamical and chemical evolution of the ISM, and hence of the galaxy as a whole. Using a 3D MHD code with adaptive mesh refinement developed for this purpose, we have investigated the rôle of magnetized matter circulation between the gaseous disk and the surrounding galactic halo. Special emphasis has been put on the effect of the magnetic field with respect to the volume and mass fractions of the different ISM "phases", the relative importance of ram, thermal and magnetic pressures, and whether the field can prevent matter transport from the disk into the halo. The simulations were performed on a grid with an area of $1 \mathrm{kpc}^{2}$, centered on the solar circle, extending $\pm 10 \mathrm{kpc}$ perpendicular to the galactic disk with a resolution as high as $1.25 \mathrm{pc}$. The simulations were run for a time scale of $400 \mathrm{Myr}$, sufficiently long to avoid memory effects of the initial setup, and to allow for a global dynamical equilibrium to be reached in case of a constant energy input rate. The main results of our simulations are: (i) The $T \leq 10^{3} \mathrm{~K}$ gas is mainly concentrated in shock compressed layers, exhibiting the presence of high density clouds with sizes of a few parsecs and $T \leq 200 \mathrm{~K}$. These structures are formed in regions where several large scale streams of convergent flow (driven by SNe) occur. They have lifetimes of a few free-fall times, are filamentary in structure, tend to be aligned with the local field and are associated with the highest field strengths; (ii) the magnetic field has a high variability and it is largely uncorrelated with the density, suggesting that it is driven by superalfvenic inertial motions; (iii) ram pressure controls the flow for $200<T \leq 10^{5.5} \mathrm{~K}$. For $T \leq 200 \mathrm{~K}$ magnetic pressure dominates, while the hot gas $\left(T>10^{5.5} \mathrm{~K}\right)$ in contrast is controlled by the thermal pressure, since magnetic field lines are swept towards the dense compressed walls; (iv) up to $49 \%$ of the mass in the disk is concentrated in the classical thermally unstable regime $200<T \leq 10^{3.9} \mathrm{~K}$ with $\sim 65 \%$ of the warm neutral medium (WNM) mass enclosed in the $500 \leq T \leq 5000 \mathrm{~K}$ gas, consistent with recent observations; (v) the volume filling factors of the different temperature regimes depend sensitively on the existence of the duty cycle between the disk and halo, acting as a pressure release mechanism for the hot phase in the disk. We find that in general gas transport into the halo in 3D is not prevented by an initial disk parallel magnetic field, but only delayed initially, for as long as it is needed to punch holes into the thick magnetized gas disk. The mean volume filling factor of the hot phase in the disk is similar in HD and MHD (the latter with a total field strength of $4.4 \mu \mathrm{G}$ ) runs, amounting to $\sim 17-21 \%$ for the Galactic supernova rate.
\end{abstract}

Key words. magnetohydrodynamics (MHD) - galaxies: ISM - ISM: evolution - ISM: bubbles - ISM: supernova remnants ISM: structure

\section{Introduction}

The evolution of galaxies is largely dependent on the physical and chemical state of the interstellar medium and thus on the rate at which they can form stars. This immediately raises the question of the structure of the ISM, i.e. the distribution of gas in so-called phases, thought to be stable regions in the $p-V$-diagram, with respect to entropy perturbations. A detailed inventory and estimate of timescales of heating and cooling processes suggested the presence of a two (Field et al. 1969) - and after the widespread interstellar OVI had been discovered - a three phase ISM (e.g. McKee \& Ostriker 1977), consisting of a cold, warm and hot component in pressure equilibrium. Since the hot intercloud medium is pervasive, it was deemed possible that it could fill a galactic corona as had been suggested by Spitzer (1956) in order to confine high velocity clouds. Observationally it was difficult to determine reliably volume filling factors for these phases in our Galaxy due to the observational vantage point. However, it became evident that the small surface coverage of HI holes in external galaxies (e.g. Brinks \& Bajaja 1986) argues for a much lower volume filling factor of the hot phase there. Since at the same time the 
extended HI (Lockman 1984) and HII (Reynolds 1985) layers of the Milky Way were discovered, it seemed plausible that break-out of supernova remnants (SNRs) was inhibited (unless they occurred at a significant height above the disk) and only the most energetic superbubbles (SBs) with at least $800 \mathrm{SNe}$ in concert (see Koo \& McKee 1992) would achieve blow-out of the disk. Things might even become worse, once a disk parallel magnetic field is considered, which according to observations should have a regular component of the order of $3 \mu \mathrm{G}$ or even higher (s. Beck 2004). If the scale height of the field is infinite, then bubbles should stall according to Tomisaka (1998) already for a moderate stellar OB association of about 50 members.

On the other hand, owing to the high sensitivity and large throughput of the ROSAT XRT (Trümper 1983) and its PSPC instrument, a number of normal spiral galaxies with soft X-ray halos were detected, e.g. NGC 891 (Bregman \& Pildis 1994) and NGC 4631 (Wang et al. 1995; Vogler \& Pietsch 1996). In some cases, even local correlations between $\mathrm{H} \alpha$, radio continuum and soft X-rays were found (see Dettmar 1992), arguing for local outflows as it had been suggested in the Galactic fountain (Kahn 1981), chimney (Norman \& Ikeuchi 1989) and the Galactic wind model (Breitschwerdt et al. 1991; Breitschwerdt \& Schmutzler 1994). However, until a few years ago it was not possible to undertake an extended 3D numerical study in order to follow the evolution of ISM structures on small and large scales simultaneously, as was done for the fountain by Avillez (1998). Recently, Korpi et al. (1999) have numerically studied the evolution of superbubbles in a magnetized disk and found that blow-out is much more likely than previously thought, mainly because bubbles evolve in an inhomogeneous background medium. Their 3D simulations are however limited by the usage of a small grid ( $1 \mathrm{kpc}$ perpendicular to the disk), so that they were able to follow only the onset of a supernova disturbed ISM for a short timescale of $\leq 100 \mathrm{Myr}$. As a consequence, these results still bear the imprint of the initial conditions, and the development of a disk-halo cycle could not be followed.

It is a fortunate coincidence that exactly 100 years after the discovery of the ISM by stationary CaII lines (Hartmann 1904), we are now for the first time in the position to give a detailed picture of the structure and evolution of the ISM on the scale of parsecs and below, that seems to converge (i.e., does not change substantially by merely increasing the numerical resolution). Our contribution here is to have carried out large scale high resolution 3D simulations of the ISM that include the Galactic magnetic field, background heating due to starlight and allow for the establishment of the duty-cycle between the disk and halo (commonly known as galactic fountain) by using a grid that extends up to $10 \mathrm{kpc}$ on either side of the midplane. Our simulations capture both the largest structures (e.g., superbubbles) together with the smaller ones (e.g., filaments and eddies) down to $1.25 \mathrm{pc}$. We investigate, among other things, the variability of the magnetic field in the Galactic disk and its correlation with the density, the rôle of ram pressure in the dynamics of disk gas and the relative weight of the ram, thermal and magnetic pressures, the mass distribution and the volume filling factors of the different temperature regimes in the ISM. Other important issues like the variation of the volume filling factors of the ISM "phases" with energy injection rate by $\mathrm{SNe}$, the dynamics of the galactic fountain, the conditions for dynamical equilibrium and the importance of convergence of these results with increasing grid resolution have been treated in Avillez (2000) and Avillez \& Breitschwerdt (2004, hereafter Paper I).

The outline of the present paper is as follows: in Sect. 2 the model and numerical setup used in the simulations is presented; Sect. 3 deals with the most important results obtained in these MHD simulations, their interpretation, and comparison with those in corresponding HD runs described in Paper I. In Sect. 4 a discussion of the results and comparison with other models is carried out. Section 5 closes the paper with a summary of the main results and some final remarks on future work.

\section{Model and simulations}

We have run high resolution kpc-scale MHD simulations of the ISM, driven by $\mathrm{SNe}$ (with a canonical explosion energy of $10^{51} \mathrm{erg}$ ) at the Galactic rate, on a Cartesian grid of $0 \leq$ $(x, y) \leq 1 \mathrm{kpc}$ size in the Galactic plane and extending $-10 \leq$ $z \leq 10 \mathrm{kpc}$ into the halo. These runs use a modified version of the model described in Avillez (2000) coupled to a threedimensional numerical AMR scheme that uses the MHD extension of the Piecewise Parabolic Method (Dai \& Woodward 1994, 1998) and the grid refinement procedure discussed in Balsara (2001) assuring that $\boldsymbol{B}$ is divergence-free during the refinement process and simulation time. The complete set of MHD equations that are solved numerically can be written in the conservative form

$\begin{array}{ll}\frac{\partial \rho}{\partial t}+\nabla \cdot \boldsymbol{\Pi}=S_{\rho} & \text { (conservation of mass), } \\ \frac{\partial \boldsymbol{\Pi}}{\partial t}+\nabla \cdot \boldsymbol{T}=S_{\Pi} \quad \text { (conservation of momentum) }, \\ \frac{\partial E}{\partial t}+\nabla \cdot \boldsymbol{U}=S_{\mathrm{E}} \quad \text { (conservation of energy), } \\ \frac{\partial \boldsymbol{B}}{\partial t}+\nabla \cdot \boldsymbol{Y}=0 \quad \text { (conservation of magnetic flux), }\end{array}$

where $\boldsymbol{\Pi}=\boldsymbol{\rho} \boldsymbol{u}$ is the mass flux, $\boldsymbol{T}=\boldsymbol{\rho} \boldsymbol{u} \boldsymbol{u}+\left(P_{\text {th }}+\frac{1}{2} B^{2}\right) \boldsymbol{I}-\boldsymbol{B} \boldsymbol{B}$ is the stress tensor, $E=\frac{1}{2} \rho u^{2}+\rho \Phi+\frac{1}{\gamma-1} P_{\text {th }}+\frac{1}{2} B^{2}$ is the total energy flux density, $\boldsymbol{U}=\left(\frac{1}{2} \rho u^{2}+\rho \Phi+\frac{\gamma}{\gamma-1} P_{\text {th }}\right) \boldsymbol{u}+B^{2} \boldsymbol{u}-u \cdot \boldsymbol{B} \boldsymbol{B}$ is the energy flux density and $\boldsymbol{Y}=\boldsymbol{u} \boldsymbol{B}-\boldsymbol{B} \boldsymbol{u}$. In these equations $\boldsymbol{B}, \boldsymbol{u}, \Phi$ and $P_{\text {th }}$ represent the magnetic field vector, velocity vector, Galactic gravitational field and the thermal pressure, respectively. The latter is related to the gas internal energy density $\left(e_{\mathrm{th}}\right)$ by $P_{\mathrm{th}}=(\gamma-1) e_{\mathrm{th}}$, with $\gamma=5 / 3 . S_{\rho}, S_{\Pi}$ and $S_{\mathrm{E}}$ are the mass, momentum and energy sources, respectively: $S_{\rho}=\frac{\Delta M_{\mathrm{SN}}}{\Delta t}$ reproduces the injection of mass per unit volume, $\Delta M_{\mathrm{SN}}$, by supernovae at instant time $\Delta t ; S_{\Pi}=-\rho \nabla \Phi+\frac{\Delta \Pi_{\mathrm{SN}}}{\Delta t}$ describes the motion of the gas in the gravitational field and the momentum injection per unit volume, $\Delta \Pi_{\mathrm{SN}}$, by $\mathrm{SNe}$ at instant $\Delta t$; and $S_{\mathrm{E}}=\Gamma(z)-\Lambda+\frac{\Delta E_{\mathrm{SN}}}{\Delta t}$ describes the energy gains due to background heating, $\Gamma(z)$, due to starlight varying with $z$ (Wolfire et al. 1995) and SNe energy injection per unit volume, $\Delta E_{\mathrm{SN}}$, at instant time $\Delta t$ and the loss of energy by radiative cooling, $\Lambda$, assuming that the gas is optically thin and in collisional ionization equilibrium. The presence of background heating leads 
here to the formation in the ISM of thermally stable regimes at $T \leq 200 \mathrm{~K}$ and $10^{3.9}<T \leq 10^{4.2} \mathrm{~K}$.

The model includes supernovae types Ia (randomly distributed in the space and having a scale height of $325 \mathrm{pc}$ ) and II (randomly distributed or clustered in OB associations formed in regions with density and temperature thresholds of $n \geq 10 \mathrm{~cm}^{-3}$ and $T \leq 100 \mathrm{~K}$, respectively). The number and masses of the OB stars are determined by an appropriate IMF following Massey et al. (1995); each star explodes after its main sequence lifetime has elapsed, using a time estimate by Stothers (1972). The trajectories of all O and B stars are followed kinematically by attributing to each a random velocity at time of formation so that the location of each exploding star is known at any time. For further details we refer to Paper I. The initial conditions comprise the setup of (i) the fixed vertical gravitational field $\Phi$ provided by the stars in the disk; of (ii) the interstellar gas having a density stratification distribution that includes the cold, cool, warm, ionized and hot "phases" in the Galaxy; and (iii) a magnetic field with uniform and random components given by $B_{\mathrm{u}}=\left(B_{\mathrm{u}, 0}\left(n(z) / n_{0}\right)^{1 / 2}, 0,0\right)$ and $\delta B=0$, respectively; here $B_{\mathrm{u}, 0}=3 \mu \mathrm{G}$ is the uniform component field strength at the Galactic midplane, $n(z)$ is the number density of the gas as a function of distance from the Galactic midplane, where it has a value of $n_{0}=1 \mathrm{~cm}^{-3}$. Periodic boundary conditions are applied along the boundaries perpendicular to the Galactic plane (i.e., the $z$-direction), while outflow boundary conditions are imposed at the top $(z=10 \mathrm{kpc})$ and bottom $(z=-10 \mathrm{kpc})$ surfaces (parallel to the galactic plane). The resolution in the grid varies on the fly according to density and/or pressure gradients thresholds with $1.25 \mathrm{pc}$ being the highest resolution of three levels of refinements, achieved in the region $|z| \leq 1 \mathrm{kpc}$, while on the rest of the domain the finest resolution is $2.5 \mathrm{pc}$ (two levels of refinement). The resolution of the coarse grid is $10 \mathrm{pc}$.

\section{Results}

\subsection{Global evolution}

The initial evolution of the disk and halo gases is characterized by the build-up of the random component of the magnetic field in addition to the establishment of the duty disk-halo-disk cycle (see Paper I). The random field component is generated during the first $15 \mathrm{Myr}$ as a result of turbulent motions mainly induced by shear flows due to $\mathrm{SN}$ explosions. For the remainder of the evolution time the random field component and the mean field oscillate around their mean values of 3.2 and $3.1 \mu \mathrm{G}$ (Fig. 1), respectively, and the average total field strength amounts to $B_{\text {tot }}=\sqrt{B_{\mathrm{u}}^{2}+(\delta B)^{2}} \simeq 4.45 \mu \mathrm{G}$. The build-up of the disk pressure by supernovae and the establishment of the duty cycle, is similar to that seen in the HD runs discussed in Paper I (see also Avillez 2000), that is, the initially stratified distribution does not hold for long as a result of the lack of equilibrium between gravity and (thermal, kinetic and turbulent) pressure during the "switch-on phase" of SN activity. As a consequence the gas in the upper and lower parts of the grid collapses onto the midplane, leaving low density material behind. However, in the MHD run it takes a longer time for the gas to descend

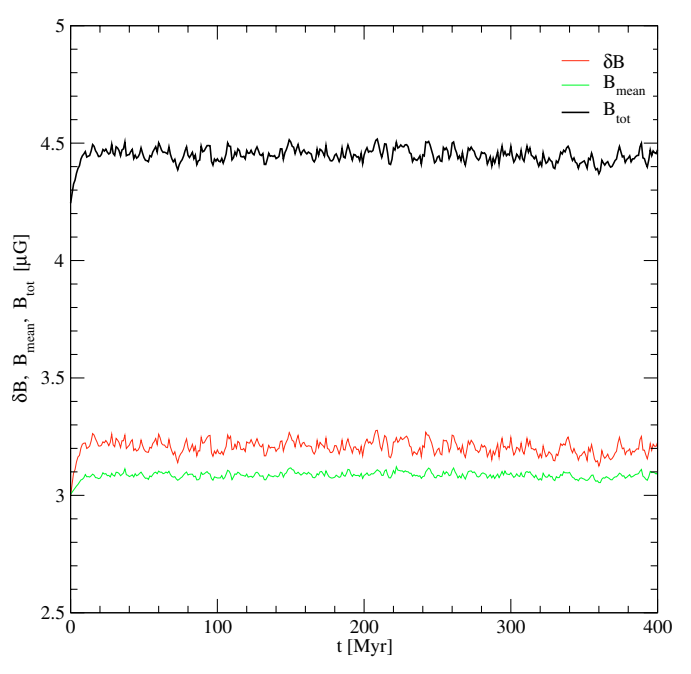

Fig. 1. Time evolution of the random field component (red), the mean field (green) and total field strength (black).

towards the disk. The complete collapse onto the midplane, like in the hydrodynamical (HD) case, is prevented as a result of the opposing magnetic pressure and tension forces. As soon as enough $\mathrm{SNe}$ have gone off in the disk building up the required pressure support, transport into the halo is not prevented, although the escape of the gas takes a few tens of Myr to occur - somewhat longer than in the pure HD case. The crucial point is that a huge thermal overpressure due to combined SN explosions can sweep the magnetic field into dense filaments and punch holes into the extended warm and ionized HI layers as seen in Fig. 2, which shows the density and magnetic field distribution in a plane perpendicular to the Galactic midplane at time $t=221.9 \mathrm{Myr}$ (the warm neutral and ionized HI gases are the light blue and greenish regions seen in the density distribution). Once such pressure release valves have been set up, there is no way from keeping the hot overpressured plasma to follow the pressure gradient into the halo. As a consequence, transport into the halo is not prevented and the duty disk-halo-disk cycle of the hot gas is fully established, in which the competition of energy input and losses into the ISM by SNe, diffuse heating, radiative cooling and magnetic pressure leads the system to evolve into a dynamical equilibrium state within a few hundred Myr (for an analytical estimate see Paper I). This time scale is considerably longer than that quoted in other papers (e.g., Korpi et al. 1999; Kim et al. 2001), because these authors do not take the galactic fountain into account. The vertical structure of the thick disk and halo is addressed in more detail in a forthcoming paper.

Figure 3 shows slices of the three-dimensional data cube of the density, temperature, pressure $P / k$, and magnetic field distributions in the Galactic midplane at $358 \mathrm{Myr}$ of evolution with a resolution of $1.25 \mathrm{pc}$. We can directly compare the morphology of the ISM between this run and the similar HD run discussed in Paper I. In both cases the highest density (and lowest temperature, if the gas had enough time to cool) gas tends to be confined into sheet-like structures (filaments in 2D) which constitute shock compressed layers (SCLs) as a result of the compression by shocks mainly driven by $\mathrm{SNe}$ in their 

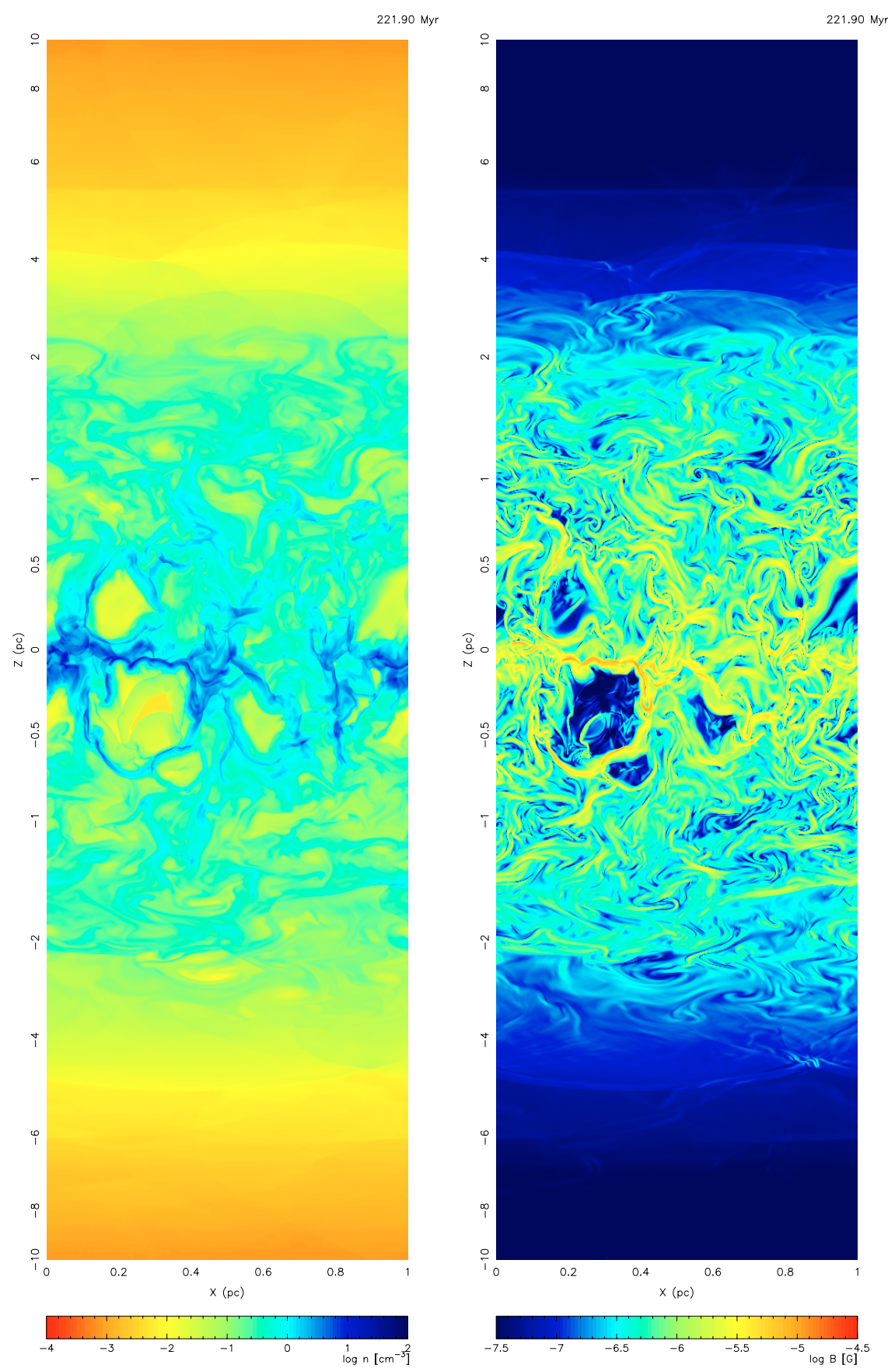

Fig. 2. Two-dimensional slices through the three-dimensional data cube showing the vertical (perpendicular to the midplane) distribution of the density (left panel) and magnetic field (right panel) at time $t=221.9 \mathrm{Myr}$. The mean field in the midplane is $3.1 \mu \mathrm{G}$. The $z$-scale above 0.5 and below $-0.5 \mathrm{kpc}$ is shrunk (in order to fit the paper size) and thus, the distribution of the labels is not uniform. Red in the density map refers to lowest density (and thus highest temperature if in pressure equilibrium), while dark blue refers to the highest density (and thus lowest temperature). In the magnetic field image red/blue correspond to the highest/lowest strength. The left panel shows the presence of a wiggly and thin disk of cold gas overlayed by a frothy thick disk composed of neutral (light blue) and ionized (greenish) gas. The expansion of superbubbles can be nicely seen, especially in the southern hemisphere. The magnetic field map shows the thin magnetized disk overlayed by loops of field lines, magnetic islands, and clouds wrapped in field lines moving downwards. From these maps it can be immediately seen that the simulations must take into account large heights on either side of the midplane, allowing for the setup of the disk-halo-disk circulation.

neighbourhood. The SCL structures are thicker in the MHD run than in the HD run, because the compression of a shock wave at given Mach number is less as energy has now to be shared between gas and magnetic field. The $T<10^{3} \mathrm{~K}$ gas in the MHD run tends to be aligned with the magnetic field, while in the HD run there is no preferred orientation. The highest densities up to $800 \mathrm{~cm}^{-3}$, observed in both runs occur in regions where several streams of convergent flow meet. The orientation of these streams is random. We note in passing, that if clouds are hit by SCLs from random directions, turbulence in the 

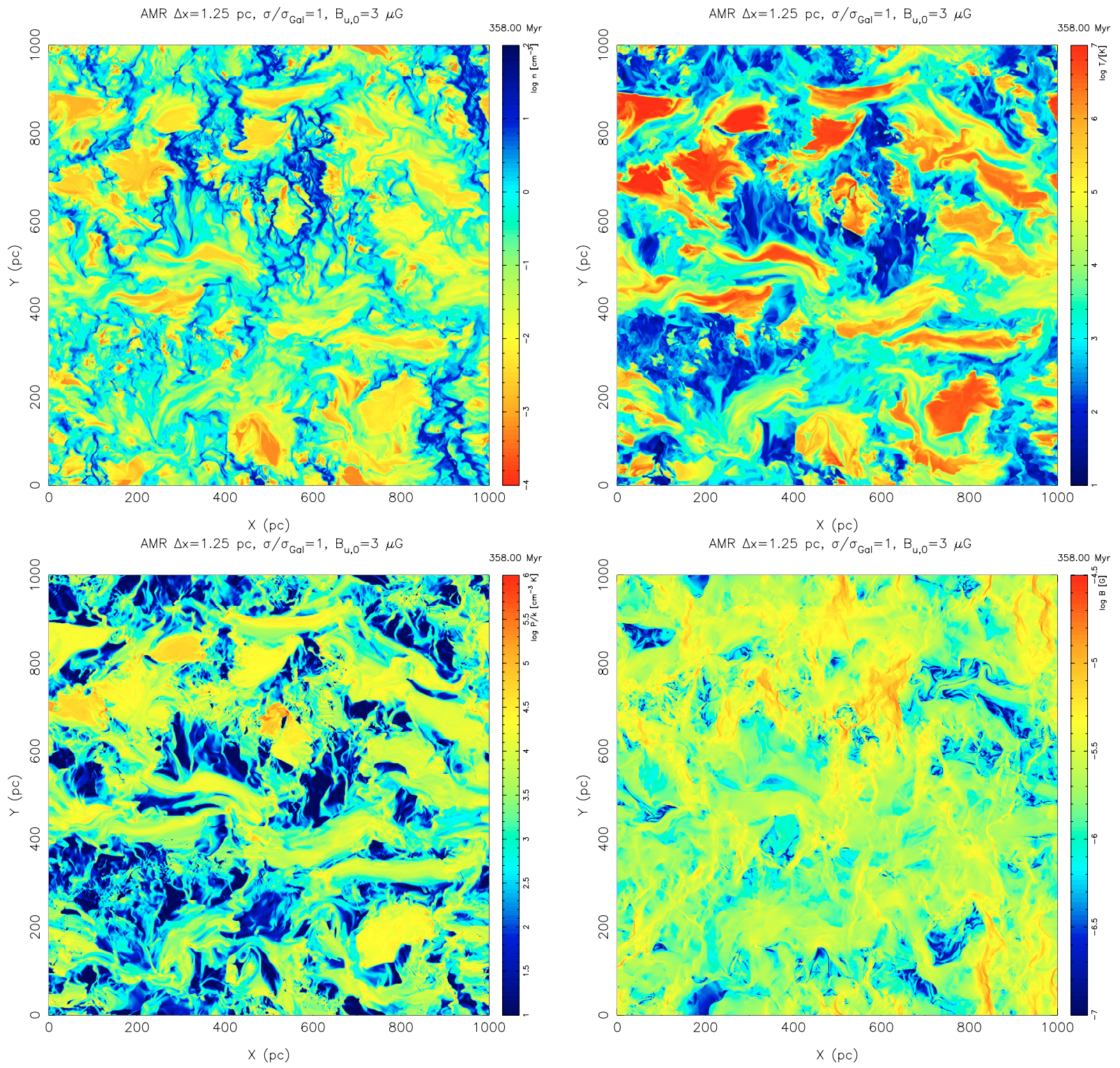

Fig. 3. Two-dimensional slices through the three-dimensional data set at $z=0$ (Galactic midplane), showing density (top left panel), temperature (top right panel), pressure $P / k$ (bottom left panel), and magnetic field (bottom right panel) after $358 \mathrm{Myr}$ of evolution. Colour bars indicate the logarithmic scale of each quantity. Red refers to lowest density, highest temperature and pressure, while dark blue refers to the highest density and lowest temperature and pressure. In the magnetic field image red/blue correspond to the highest/lowest strength. Direct visual inspection shows a strong inverse spatial correlation between gas density and temperature (although the pressure diagram is still far from uniform), whereas the correlation between magnetic field strength and gas density is only present for the cold dense regions. For higher temperature regions this correlation becomes rather poor and vanishes completely for the hot gas (s. Fig. 4).

interior is generated. Tapping the turbulent ISM energy reservoir, which is huge, represents a neat way of sustaining supersonic turbulence inside molecular clouds, which as it is known, dissipates very efficiently. We will explore this idea in more detail in a forthcoming paper.

In the MHD run cold denser regions are dominated by the magnetic field and are more filamentary in structure than low density regions (which have low field strengths, although, as seen in Fig. 4, a wide range of $B$-field strengths is spanned) due to the anisotropy introduced by the strong field, which is much better correlated with the gas density for lower than for higher temperature gas.

\subsection{Evolution of supernova remnants and superbubbles in the magnetized ISM}

The evolution of magnetized SNRs and SBs has been extensively studied in the literature (see e.g. Kulsrud et al. 1965; Summers 1975; Guiliani 1982). In particular the question of break-out has been addressed in numerical studies by Tomisaka (1990) and Mineshige et al. (1993), who both come to the conclusions that bubbles could be confined to the gaseous disk. Since this is in disagreement with our simulations we will discuss the subject of SB evolution (which includes SNRs as a 


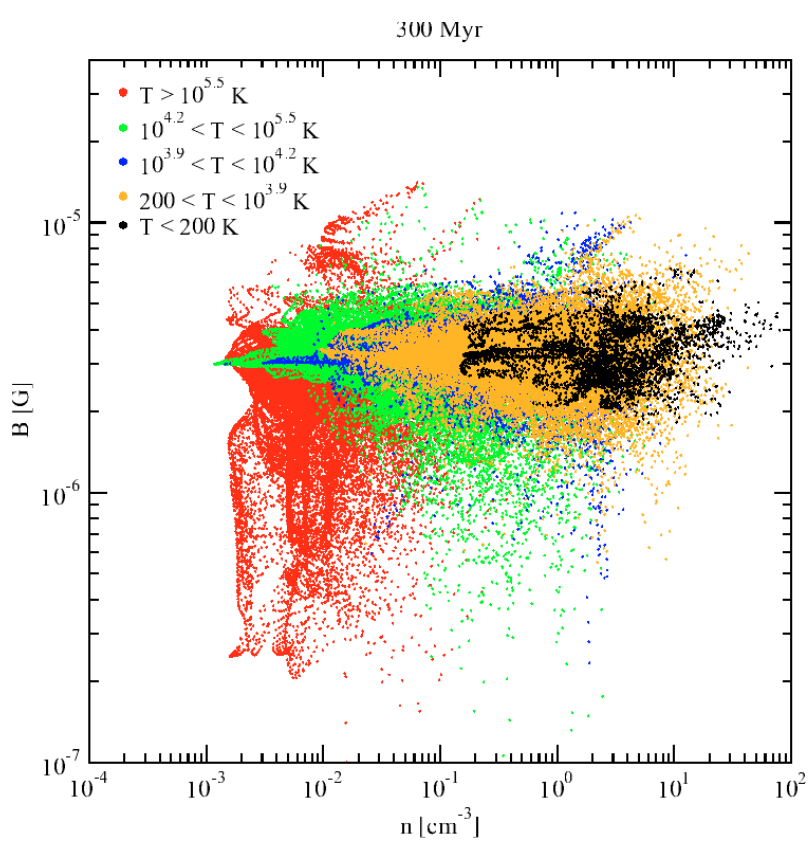

Fig. 4. Scatter plot of $B$ versus $\rho$ for $T \leq 200$ (black), $200<T \leq 10^{3.9}$ (orange), $10^{3.9}<T \leq 10^{4.2}$ (blue), $10^{4.2}<T \leq 10^{5.5} \mathrm{~K}$ (green), and $T>10^{5.5} \mathrm{~K}$ (red) regimes at $300 \mathrm{Myr}$ of disk evolution. The points in the plot are sampled at intervals of four points in each direction. Note that during the evolution of the system the field strength broadened its distribution spanning two orders of magnitude after $300 \mathrm{Myr}$.

special case) in an ambient medium with frozen-in magnetic field in more detail.

During their initial expansion phase, young supernova remnants are roughly spherical as long as the radius is below or at the order of the magnetic field curvature scale, but as time progresses magnetic tension forces increase as the radius of curvature of the field lines decreases as $F_{\mathrm{t}} \sim B_{\mathrm{u}}^{2} /\left(4 \pi R_{\mathrm{c}}\right)$, where $R_{\mathrm{c}}$ and $F_{\mathrm{t}}$ are the radius of field curvature and tension force, respectively, and $B_{\mathrm{u}}$ is the uniform field component. It is straightforward to estimate the average radius of a superbubble, or respectively the time, when the shell stalls, because in the long run magnetic tension forces beat the thermal pressure force in the hot interior, since tension increases and pressure decreases with time. This argument assumes some kind of ideal configuration, e.g. that the shock normal is perpendicular to the magnetic field direction, which as can be seen in the simulations, does in general not exist, as the field is tangled and inhomogeneous. It should also be kept in mind that such an analytic analysis ignores the possibility of MHD instabilities that would promote mixing with the ambient medium and escape of hot bubble gas.

In the following we assume that the outer shell will have cooled off and collapsed into a thin shell before slowing down considerably, being subject to magnetic and thermal pressure. Let $R_{\mathrm{S}}$ be the shell radius, $\theta$ be the polar angle between the radial and the magnetic field direction $\boldsymbol{B}$, and $\delta R$ the shell thickness, so that the bubble radius is given by $R_{\mathrm{b}}=R_{\mathrm{s}}-\delta R$. For $\theta=0$ the outer shock is purely gasdynamic, as compression along the $B$-field is unimpeded. However flux conservation causes the field to wrap around the bubble and carry a tangential component as $\theta$ increases. The bending of the lines of force near the polar region leads to a deflection of the incoming flow and thus to a $\theta$-dependent momentum transport. As this reduces the inertia of the shell near the polar region, the deceleration there is more effective leading to a "dimple" (s. Ferrière et al. 1991). Whereas the pressure is purely thermal here, the magnetic pressure increases with $\theta$ and will dominate completely for $\theta \rightarrow \pi / 2$. Flux conservation at co-latitude $\theta$ in the thin shell approximation requires

$$
\begin{aligned}
\int \boldsymbol{B} \mathrm{d} \boldsymbol{f} & =\pi R_{\mathrm{s}}^{2} \sin ^{2} \theta B_{0}=\int_{R_{\mathrm{b}}}^{R_{\mathrm{s}}} 2 \pi R \mathrm{~d} R B_{\theta} \sin \theta \\
& =\pi\left(R_{\mathrm{s}}^{2}-R_{\mathrm{b}}^{2}\right) B_{\theta} \sin \theta,
\end{aligned}
$$

and since $R_{\mathrm{b}}^{2}=\left(R_{\mathrm{S}}-\delta R\right)^{2} \approx R_{\mathrm{S}}^{2}-2 R_{\mathrm{s}} \delta R$, we obtain

$B_{\theta} \approx \frac{1}{2} B_{0} \frac{R_{\mathrm{s}}}{\delta R} \sin \theta$

which at the equatorial region, where field tension is strongest, yields $B_{\theta}=\frac{1}{2} B_{0} \frac{R_{\mathrm{s}}}{\delta R}$, where $B_{\theta}$ is the poloidal field component in the shell.

Numerical simulations show (e.g. Ferrière et al. 1991) that in case of interstellar magnetic field strengths of the order 3-5 $\mu \mathrm{G}$ the evolution until the outer shock decays into a fast magnetosonic wave can still be well described by a similarity solution (assuming a homogeneous ambient medium) given by McCray \& Kafatos (1987). In the case of quasi-sphericity, the momentum equation for self-similar flow, and using Eq. (6), reads

$$
\begin{aligned}
\frac{\mathrm{d}}{\mathrm{d} t}\left(M_{\mathrm{s}} \dot{R}_{\mathrm{s}}\right) & =4 \pi\left(R_{\mathrm{s}}^{2} P_{\mathrm{b}}-\frac{B_{\theta}^{2}}{4 \pi} R_{\mathrm{s}} \delta R\right) \\
& =4 \pi R_{\mathrm{s}}^{2}\left(P_{\mathrm{b}}-P_{m 0} \frac{R_{\mathrm{s}}}{2 \delta R}\right) .
\end{aligned}
$$

Here $P_{\mathrm{b}}$ and $P_{m 0}=\frac{B_{0}^{2}}{8 \pi}$ are the thermal pressure in the bubble and the magnetic pressure in the ambient medium, respectively. The bubble expansion will start to reverse, once the force acting on the shell points inwards. In other words, the radius or time of maximum extension of the bubble in the equatorial direction can be roughly estimated by demanding that the term in brackets on the right-hand side of Eq. (7) vanishes, giving

$P_{\mathrm{b}}=P_{m 0} \frac{R_{\mathrm{s}}}{2 \delta R}$

The applicability of similarity solutions (cf. Weaver et al. 1977) provides $P_{\mathrm{b}}$ and $R_{\mathrm{s}}$ by

$$
\begin{aligned}
R_{\mathrm{S}} & =A\left(\frac{L_{*}}{\rho_{0}}\right)^{1 / 5} t^{3 / 5} \\
A & =\left(\frac{125}{154 \pi}\right)^{1 / 5} \\
P_{\mathrm{b}} & =D L_{*}^{2 / 5} \rho_{0}^{3 / 5} t^{-4 / 5} \\
D & =\frac{7}{(3850 \pi)^{2 / 5}}
\end{aligned}
$$

with $L_{*}$ denoting the mechanical luminosity generated by stellar wind and supernova activity of the central star cluster, and 
$\rho_{0}=$ const. is the ambient mass density. Since SNe are the dominant source for rich clusters one can use for a simple estimate, $L_{*}=N_{*} E_{\mathrm{SN}} / \tau_{\mathrm{MS}}=3.17 N_{*} E_{51} / \tau_{7} \times 10^{36} \mathrm{erg} \mathrm{s}^{-1}$, where $N_{*}$ is the number of supernovae, $E_{\mathrm{SN}}$ and $E_{51}, \tau_{\mathrm{MS}}$ and $t_{7}$ are the supernova energy, also measured in $10^{51} \mathrm{erg}$, respectively, and the main sequence life time of the least massive star, also in units of $10 \mathrm{Myr}$, respectively.

To solve Eq. (8) we need an expression for the relative shell thickness, $\delta R$. In the equatorial region, the pressure in the shell at later times will be dominated by magnetic pressure so that momentum conservation across the shock requires

$\rho_{0} \dot{R}_{\mathrm{s}}^{2} \simeq \frac{B_{\theta}^{2}}{8 \pi}$

and using Eq. (6)

$$
\begin{aligned}
\dot{R}_{\mathrm{s}}^{2} & =\frac{B_{0}^{2}}{32 \pi \rho_{0}}\left(\frac{R_{\mathrm{s}}}{\delta R}\right)^{2} \sin ^{2} \theta \\
\Rightarrow \frac{\delta R}{R_{\mathrm{s}}} & =\frac{1}{2 \sqrt{2}} \frac{V_{\mathrm{A} 0}}{\dot{R}_{\mathrm{s}}} \sin \theta,
\end{aligned}
$$

implicitly assuming that the shock propagates in an undisturbed ambient medium, with $V_{\mathrm{A} 0}=\frac{B_{0}}{\sqrt{4 \pi \rho_{0}}}$ being the Alfvén speed there. Equation (12) shows the thickening of the shell with time as it decelerates, like $\delta R \propto R_{\mathrm{s}} / \dot{R}_{\mathrm{s}} \propto t$ :

$$
\begin{gathered}
\delta R=\frac{5}{6 \sqrt{2}} V_{\mathrm{A} 0} t \sin \theta \\
\Leftrightarrow \delta R=1.2 \frac{B_{-6} t_{6}}{\sqrt{n_{0}}} \sin \theta \mathrm{pc},
\end{gathered}
$$

where $n_{0}, B_{-6}$ and $t_{6}$ denote the ambient number density in units of $\mathrm{cm}^{-3}$, the ambient magnetic field strength in units of $\mu \mathrm{G}$, and the time in Myr, respectively.

As long as the ambient thermal pressure is small compared to the pressure inside the bubble and the magnetic pressure in the shell, and combining Eqs. (10)-(12), and inserting them into Eq. (8), we obtain for the time $t_{\max }$ of maximum bubble expansion (for $\theta=\pi / 2$, where tension forces are strongest)

$$
\begin{aligned}
t_{\max } & =\kappa\left(\frac{L_{*} \rho_{0}^{3 / 2}}{B_{0}^{5}}\right)^{1 / 2} \\
\kappa & =\left(\frac{5}{3} \frac{D}{A}\right)^{5 / 2}(8 \pi)^{5 / 4} \\
& =4.255 \\
\Rightarrow t_{\max } & =718.39 L_{37}^{1 / 2} n_{0}^{3 / 4} B_{-6}^{-5 / 2} \mathrm{Myr} ;
\end{aligned}
$$

here $L_{37}$ denotes the mechanical luminosity in units of $10^{37} \mathrm{erg} \mathrm{s}^{-1}$. Note, that this is also roughly the time scale on which the outer shock decays into a fast magnetosonic wave, which is disturbing the ambient medium with a characteristic speed of $a_{\mathrm{f}}=\sqrt{\frac{k_{\mathrm{B}} T_{0}}{\mu \bar{m}}+\frac{B_{0}^{2}}{4 \pi \rho_{0}}}$, with $k_{\mathrm{B}}, \mu, \bar{m}$ and $T_{0}$ denoting Boltzmann's constant, the mean molecular weight, the mean particle mass of the gas and the ambient temperature, respectively. Equating this to the shock speed travelling outwards at $\dot{R}_{\mathrm{s}}$, results in a typical decay time of $t_{\mathrm{f}} \simeq$ $285 L_{37}^{1 / 2} n_{0}^{3 / 4} B_{-6}^{-5 / 2}\left(0.58+\frac{T_{3} n_{0}}{\mu B_{-6}^{2}}\right)^{-5 / 4}$ Myr. Here $T_{3}$ denotes the ambient temperature in units of $10^{3} \mathrm{~K}$. For example, taking a field strength of $B_{-6}=5$, an ambient density of $n_{0}=0.3$ and $L_{37}=3$, corresponding to a cluster with about $50 \mathrm{SNe}$, we obtain $t_{\mathrm{f}} \simeq 6.2 \mathrm{Myr}$. Since the dependence on $B_{0}, n_{0}$, and $L_{*}$ is exactly the same as in Eq. (18), the outer shock in the equatorial region will always have decayed just about before the bubble will stall. Although several of the assumptions made above will break down, the qualitative picture will be approximately correct. In particular, the shell will be still small compared to the bubble radius, i.e. $\frac{\delta R}{R_{\mathrm{s}}}=\frac{1}{2 \sqrt{2}} \frac{V_{A 0}}{a_{\mathrm{f}}}=\frac{1}{2}(2+\beta)^{-1 / 2}$, where $\beta=8 \pi n_{0} k_{\mathrm{B}} T_{0} / B_{0}^{2}$ is the plasma beta in the ambient medium. Thus even for $\beta \rightarrow 0, \delta R \leq 0.35 R_{\mathrm{s}}$.

The radius of maximum expansion (perpendicular to the $B$-field direction) is therefore given by

$$
\begin{aligned}
\Rightarrow R_{\max } & =\left(\frac{125 \kappa^{3}}{154 \pi}\right)^{1 / 5} L_{*}^{1 / 2} \rho_{0}^{1 / 4} B_{0}^{-3 / 2} \\
& =2217 L_{37}^{1 / 2} n_{0}^{1 / 4} B_{-6}^{-3 / 2} \mathrm{pc} .
\end{aligned}
$$

The maximum extension therefore depends most sensitively on the magnetic field strength, and only weakly on the ambient density. Using the above values for $B_{0}, n_{0}$ and $L_{37}$, we obtain $R_{\max } \approx 254 \mathrm{pc}$ and $t_{\max } \approx 9 \mathrm{Myr}$, well within our grid size and simulation timescale, respectively. With density inhomogeneities and stratification perpendicular to the disk left aside, the bubble is still not too aspherical. In the following however, the contraction in the equatorial region will make it more elongated towards the poles, i.e. along the magnetic field. In addition, contraction will increase the pressure in the interior thus acting against the tension forces, but also enhance expansion predominantly along the field. We therefore conclude that elongated features in our simulations correspond to old superbubbles, unless large density gradients in the ambient medium have been present before. If the latter is the case, then the bubbles continue early on to expand into the neighbouring regions where the field lines have been moved aside by other bubbles. In any case, the evolved bubbles lose their spherical form acquiring an oval-like appearance (see Fig. 3). After $358 \mathrm{Myr}$ of evolution there are still a large number of regions were the mean field maintains its initial orientation, although locally it is heavily distorted by $\mathrm{SNe}$ (Fig. 3). Interestingly, the average $P / k$ value is higher in the MHD case due to the magnetic field contribution, which in the absence of dissipative processes and loss of flux always is present.

Perpendicular to the galactic plane the expansion of bubbles will be alleviated due to the density stratification of the extended disk and Rayleigh-Taylor instabilities can occur once the shell accelerates over a few density scale heights. The effect of the magnetic field then will be similar to surface tension in an ordinary fluid, limiting the growth of the most unstable modes to a maximum wave number. It is therefore expected that shell fragments or cloudlets should have a minimum size. A number of shells will stall before that happens, and these will appear in simulations and observations, like e.g. NGC 4631 (Wang et al. 2001) as extended loops. 


\subsection{Field dependence with density}

During the evolution of the system, thermal and dynamical processes broaden the distribution of the field strength with density so that after the global dynamical equilibrium has been set up, the field strength in the disk spans two orders of magnitude from 0.1 to $15 \mu \mathrm{G}$ (see Fig. 4). The spreading in the field strength increases with temperature, being largest for the hot $\left(T>10^{5.5} \mathrm{~K}\right.$ ) and smallest (between 2 and $6 \mu \mathrm{G}$ ) for the cold $(T \leq 200 \mathrm{~K})$ gas. The lowest $(0.1 \mu \mathrm{G})$ and higher (larger than $10 \mu \mathrm{G})$ field strengths are associated with $T>10^{4.2}$ gas and densities of $0.1-1 \mathrm{~cm}^{-3}$. The intermediate temperature regimes have the bulk of their scatter points similarly distributed between 2 and $6 \mu \mathrm{G}$, although there is a clear distinction between the different temperature regimes with respect to the density coverage. It can be seen that the $10^{4.2}<T \leq 10^{5.5} \mathrm{~K}$ gas covers a large fraction of the scatter points overlapping with the coolest and partially with the hot regimes, and there, mainly with those of low densities and high field strengths. It almost completely covers points located in the $10^{3.9}<T \leq 10^{4.2} \mathrm{~K}$ both in the field strength and in the density distribution. The thermally unstable gas $\left(200<T \leq 10^{3.9} \mathrm{~K}\right)$ completely overlaps the $T \leq 200 \mathrm{~K}$ scatter points, although the latter covers smaller field strength intervals than the unstable gas. The $10^{4.2}<T \leq 10^{5.5}$ gas coverage with other (stable) regimes is an indication that it is in a transient state trying to move towards thermally stable regions at $T \leq 10^{4.2} \mathrm{~K}$ or to the $T>10^{5.5} \mathrm{~K}$ range. Whether this gas becomes part of a classically stable region, however, depends if it has enough time to cool down (or to be heated up) with respect to dynamical time scales, which change its location on the $p-V$-diagram. The higher field strengths at the intermediate temperature regimes are related to the increase in compression of the field lines in newly formed shells and SCLs.

The large scatter in the field strength for the same density, seen in Fig. 4, suggests that the field is being driven by the inertial motions, rather than it being the agent determining the motions. In the latter case the field would not be strongly distorted, and it would direct the motions predominantly along the field lines. In ideal MHD field diffusion is negligible, and the coupling between matter and field should be perfect (we are of course aware of numerical diffusion, which weakens the argument at sufficiently small scales). Therefore gas compression is correlated with field compression, except for strictly parallel flow. The high field variability is also seen in the right bottom panel of Fig. 3, which shows a highly turbulent field, that seems to be uncorrelated with the density, and thus, the classical scaling law $B \sim \rho^{\alpha}$, with $\alpha=1 / 2$, according to the Chandrasekhar-Fermi (CF) model (1953) will not hold. It should be kept in mind that in CF it was assumed that the field is distorted by turbulent motions that were subalfvénic, whereas in our simulations in addition both supersonic and superalfvénic motions can occur, leading to strong MHD shocks. In other words, according to the CF model, the turbulent velocity is directly proportional to the Alfvén speed, which in a SN driven ISM need not be the case. If ram pressure fluctuations in the ISM are dominant as our simulations suggest, we would indeed not expect a perfect correlation with $\alpha=1 / 2$

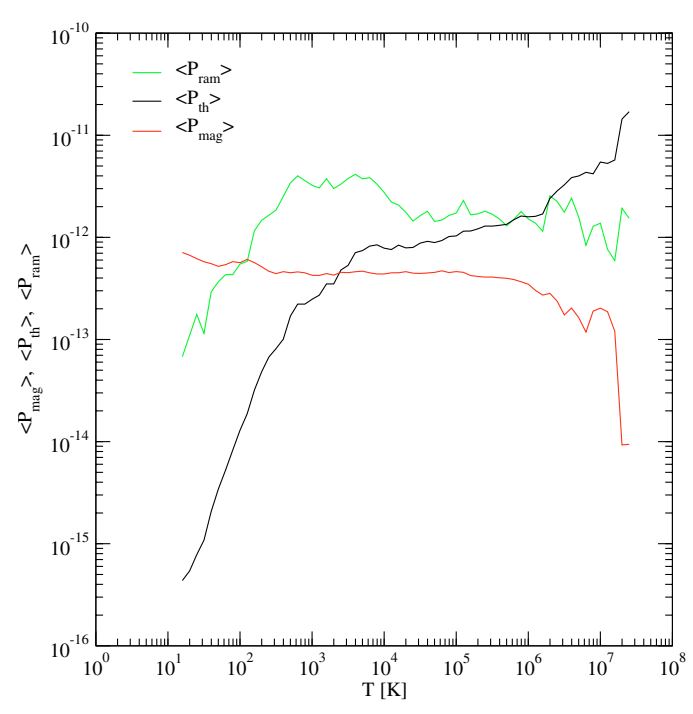

Fig. 5. Comparison of the averages $\left\langle P_{\text {ram }}\right\rangle$ (green), $\left\langle P_{\text {th }}\right\rangle$ (black), and $\left\langle P_{\text {mag }}\right\rangle$ (red) as function of the temperature (in the simulated disk $|z| \leq$ $250 \mathrm{pc}$ ) averaged over temperature bins of $\Delta \log T=0.1 \mathrm{~K}$.

but a broad distribution of $B$ versus $\rho$. Although in general $0 \leq \alpha \leq 1$ would be expected, it should be noted that in reality heating and cooling processes, and even magnetic reconnection could induce further changes, making the correlation rather complex.

\subsection{Weight of thermal, magnetic and ram pressures}

In order to gain insight into the driving forces in the simulated disk $(|z| \leq 250 \mathrm{pc})$ we turn now to the relative weight of the ram, thermal and magnetic pressures as function of the temperature averaged over bins of $\Delta \log T=0.1 \mathrm{~K}$ (Fig. 5). The average distributions shown in the figure have been calculated by using 51 snapshots with a time interval of 1 Myr during the time evolution of 350 and 400 Myr.

The relative importance of thermal pressure over ram and magnetic pressures increases with temperature. The gas with $T \geq 10^{5.5} \mathrm{~K}$ is mainly thermally driven, while at lower temperatures thermal pressure has no influence on the dynamics of the flow. For $T \leq 150 \mathrm{~K}, P_{\text {th }}<P_{\text {ram }}<P_{\text {mag }}$ indicating that the gas is dominated by the Lorentz $\boldsymbol{j} \times \boldsymbol{B}$ forces, and the magnetic field determines the motion of the fluid. At the intermediate temperatures ram pressure dominates, and therefore, the magnetic pressure does not act as a significant restoring force (see Passot \& Vázquez-Semadeni 2003) as it was already suggested by the lack of correlation between the field strength and the density. It is also noteworthy that in this temperature range $\left(150-7.4 \times 10^{5} \mathrm{~K}\right)$ the weighted magnetic pressure is roughly constant, suggesting that the magnetic and thermal pressures are largely independent, whereas both thermal and ram pressures undergo large variations in this temperature interval.

We therefore conclude that in galactic disk environments the magnetic field will have a strong influence on the cold gas, particularly by controlling cloud and star formation processes. On the other hand, the warm and hot phases of the ISM, which are dominating by volume but not by mass, are affected 
dynamically only to a minor extent by magnetic pressure forces. However, it should be kept in mind, that microscopic processes, like heat conduction, will be strongly modified by the presence of a magnetic field.

\subsection{Thermal and magnetic pressures}

The averaged (over the period 350-400 Myr) volume weighted histograms of the thermal, magnetic and total $\left(P_{\mathrm{tot}}=P_{\mathrm{th}}+P_{\mathrm{mag}}\right.$, which is calculated at each grid cell, and then used in the construction of the corresponding histogram) pressures of the different temperature regimes in the magnetized ISM (see Fig. 6) show that for volume fractions $\mathrm{d} N / N \geq 10^{-4}$ the full distributions (shown by the dashed lines) have a pressure coverage running from three (for $P_{\text {tot }}$ ) to more than five (for $P_{\text {th }}$ ) orders of magnitude and having smooth (for $P_{\mathrm{th}}$ ) and steep (for $P_{\mathrm{mag}}$ and $\left.P_{\text {tot }}\right)$ peaks located in the $\left[2 \times 10^{-13}, 2 \times 10^{-12}\right]$ dyne $\mathrm{cm}^{-2}$ interval. These peaks are determined by the contributions of the $200<T \leq 10^{3.9} \mathrm{~K}$ and $10^{4.2}<T \leq 10^{5.5} \mathrm{~K}$ regimes followed by the hot gas. The thermally stable regimes $10^{3.9}<$ $T \leq 10^{4.2} \mathrm{~K}$ and $T \leq 200 \mathrm{~K}$, by this order, have the smallest contribution to the peaks.

In the thermal and magnetic pressure histograms it is possible to distinguish two regions $\left(P_{\text {th }}<10^{-13}\right.$ and $P_{\text {th }}>$ $10^{-12}$ dyne $\left.\mathrm{cm}^{-2}\right)$, which are determined by the gas with $T \leq 10^{3.9}$ and $T>10^{4.2} \mathrm{~K}$ (with the hot gas being the main contributor for the high pressure region), respectively. In the $P_{\text {mag }}$ histogram (that has a conical structure covering the $\left[10^{-15}, 5 \times 10^{-12}\right]$ dyne $\mathrm{cm}^{-2}$ region for $\mathrm{d} N / N \geq 10^{-4}$ ) all regimes contribute to these two regions, although the hot gas is the main contributor to the low pressure region and the cold gas contributes mainly to the high pressure range.

A comparison between the top and middle panels of the figure indicates that the highest values of the total pressure, i.e., $P_{\text {tot }} \geq 10^{-12}$ dyne $\mathrm{cm}^{-2}$, are mainly of thermal origin. Contributions come from the gas with $T>10^{4.2} \mathrm{~K}$ and in particular from the hot gas (whose histogram has a central peak located around $2 \times 10^{-12}$ dyne $\mathrm{cm}^{-2}$ and two smaller peaks in the right wing of the histogram at $P_{\text {th }}>10^{-11}$ dyne $\mathrm{cm}^{-2}$, resulting from $\mathrm{SN}$ events). The gas with $200<T \leq 10^{4.2}$ has a very small contribution and the cold gas practically none. For the remaining pressure range $\left(P_{\text {tot }}<10^{-12}\right.$ dyne $\left.\mathrm{cm}^{-2}\right)$ both magnetic and thermal pressures contribute, with the magnetic contributions having a larger weight than the thermal ones.

\subsection{Volume weighted histograms}

In order to gain insight into the distribution of the disk gas as a function of density or temperature, it is useful to construct volume weighted histograms. They give us information about the occupation fraction of a certain "gas phase" with respect to volume averaged density and temperature. Therefore a peak in the histogram tells us, at which density/temperature a particular phase is concentrated, because here the occupation fraction is highest. A comparison between the averaged (over the time 350 through $400 \mathrm{Myr}$ ) volume weighted histograms of the density and temperature of the magnetized and unmagnetized disk gas
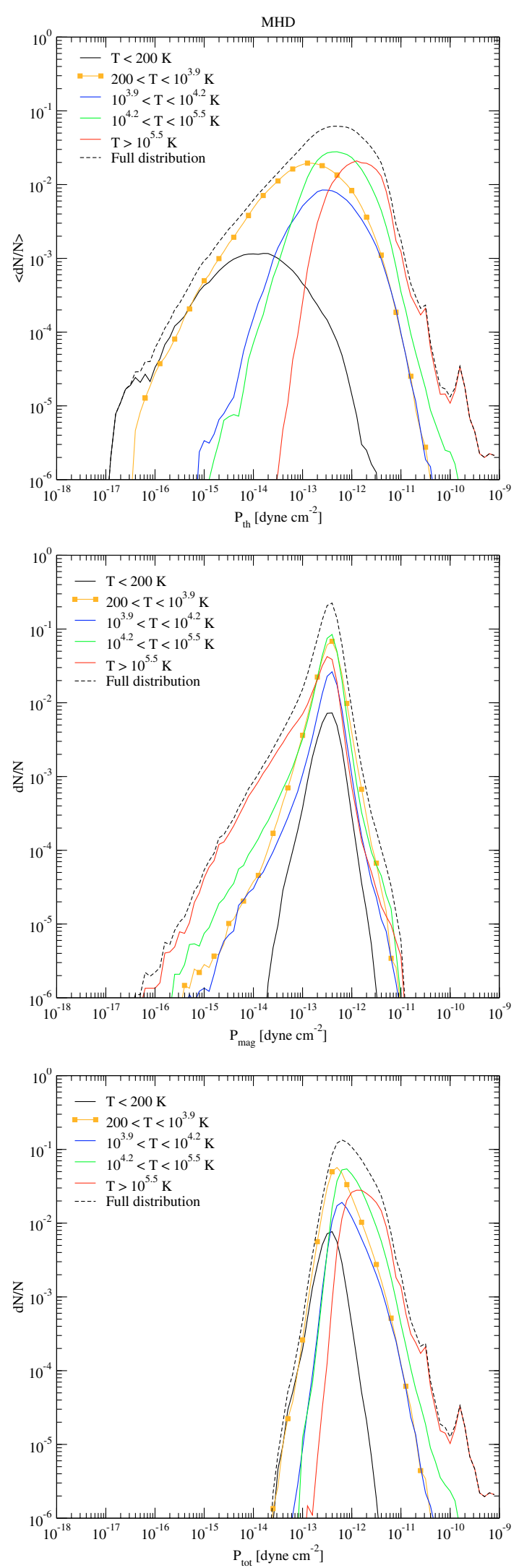

Fig. 6. Averaged volume weighted histograms of the thermal (top panel), magnetic (middle panel) and total (bottom panel) pressures for the different temperature regimes in the ISM. The total distribution of each pressure is shown by the dashed black line. The histograms have been calculated using 51 snapshots with a time interval of $1 \mathrm{Myr}$ between 350 and 400 Myr. 

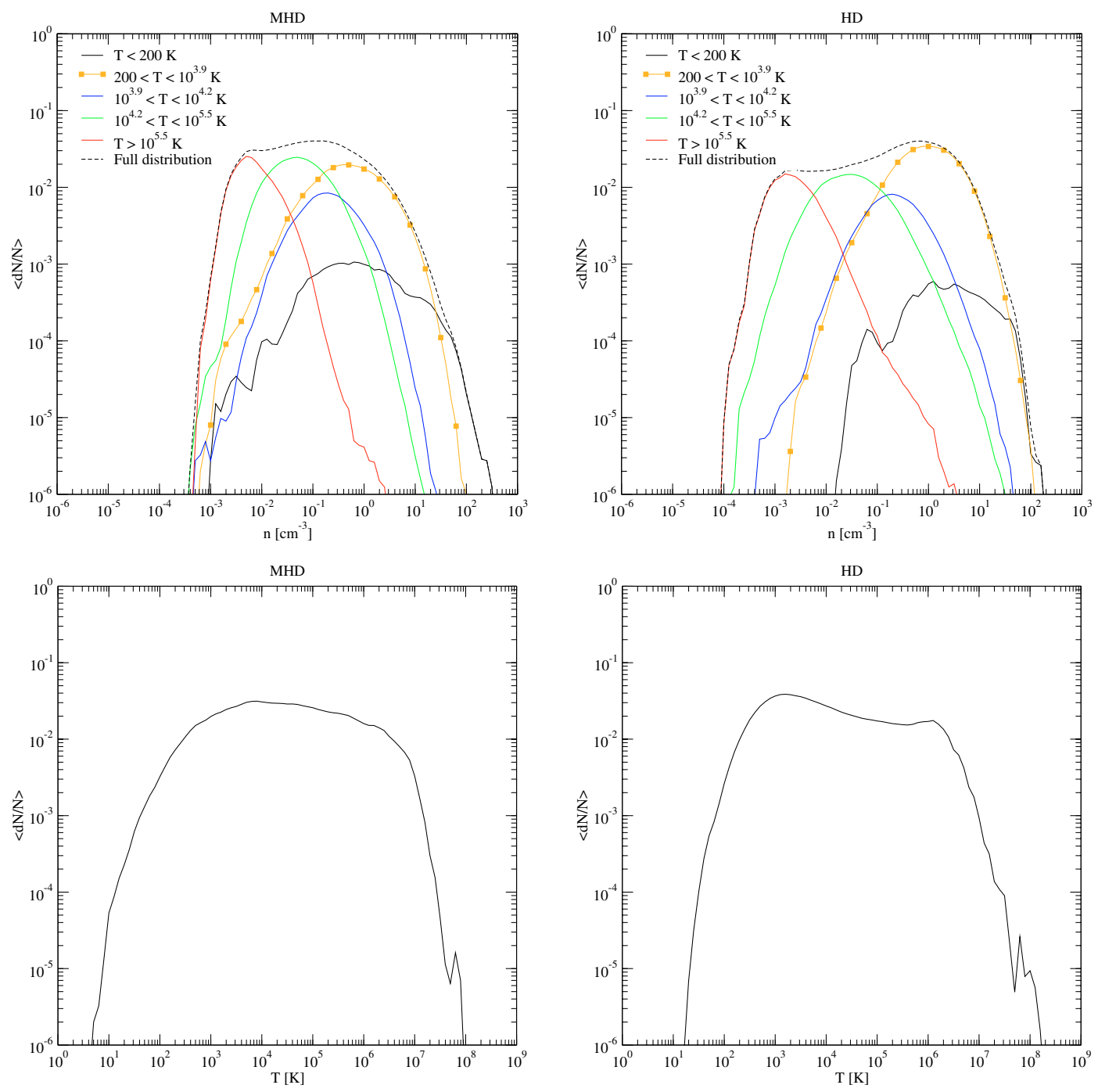

Fig. 7. Averaged volume weighted histograms of the density for the different temperature regimes of the disk gas (the full distribution is shown by the dashed line) in the top panels and averaged temperature histograms in the bottom panels for the MHD run (left column) and the HD run (right column). The histograms have been calculated using 51 snapshots with a time interval of $1 \mathrm{Myr}$ between 350 and $400 \mathrm{Myr}$.

(Fig. 7) shows noticeable differences that include the (i) decrease/increase by an almost order of magnitude in the histograms density/temperature coverage; (ii) change in the relative weight of the dominant temperature regimes (in the density histograms) and consequently; (iii) changes in the pronounced bimodality of the total density and temperature histograms.

The density histograms in both HD and MHD runs are dominated by the $T>10^{5.5} \mathrm{~K}$ (less important), $10^{4.2}<T \leq$ $10^{5.5} \mathrm{~K}$ and $200<T \leq 10^{3.9} \mathrm{~K}$ regimes. The latter regime is the most important in the HD run contributing to the pronounced peak around $n \sim 1 \mathrm{~cm}^{-3}$ in the total density distribution, whereas in the magnetized ISM the latter two regimes interchange in importance (there is a strong reduction in the peak of the $200<T \leq 10^{3.9} \mathrm{~K}$ gas). This leads to a shift of the peak in the total density histogram to $n \sim 0.2 \mathrm{~cm}^{-3}$, where it is mainly dominated by the $10^{4.2}<T \leq 10^{5.5} \mathrm{~K}$ gas contribution. The hot gas averaged PDFs have a similar shape but different density coverage, being larger in the HD run by an order of magnitude and peak location (being a factor of two higher in the MHD run). This suggests that the areas underneath the histograms, that is, the volume filling factors of the hot gas, are not much different in the two runs. The smallest peaks seen in the density histograms belong to the thermally stable regimes $T \leq 200 \mathrm{~K}$ (which has the smallest occupation fraction), followed by the $10^{3.9}<T \leq 10^{4.2} \mathrm{~K}$ regime. With the inclusion of the magnetic field, there is an increase in the density coverage by the cold gas histogram and thus of its occupation fraction in the disk.

The variations seen in the density weighted histograms are also seen in temperature PDFs having different structures. In effect, while the temperature PDF of the HD run has a bimodal structure (as it has two peaks: one at $2000 \mathrm{~K}$ and another around $10^{6} \mathrm{~K}$ ), in the MHD run the decrease/increase in importance of the $10^{3.9}<T \leq 10^{4.2} \mathrm{~K} / 10^{4.2}<T \leq 10^{5.5} \mathrm{~K}$ leads to the reduction/increase of the occupation fraction of these regimes and therefore to a change of the histogram structure appearing it to be unimodal. This variation of the intermediate region appears to be an effect of the presence of the magnetic field, with the smoothing effect being less pronounced for lower field strengths in the disk. 

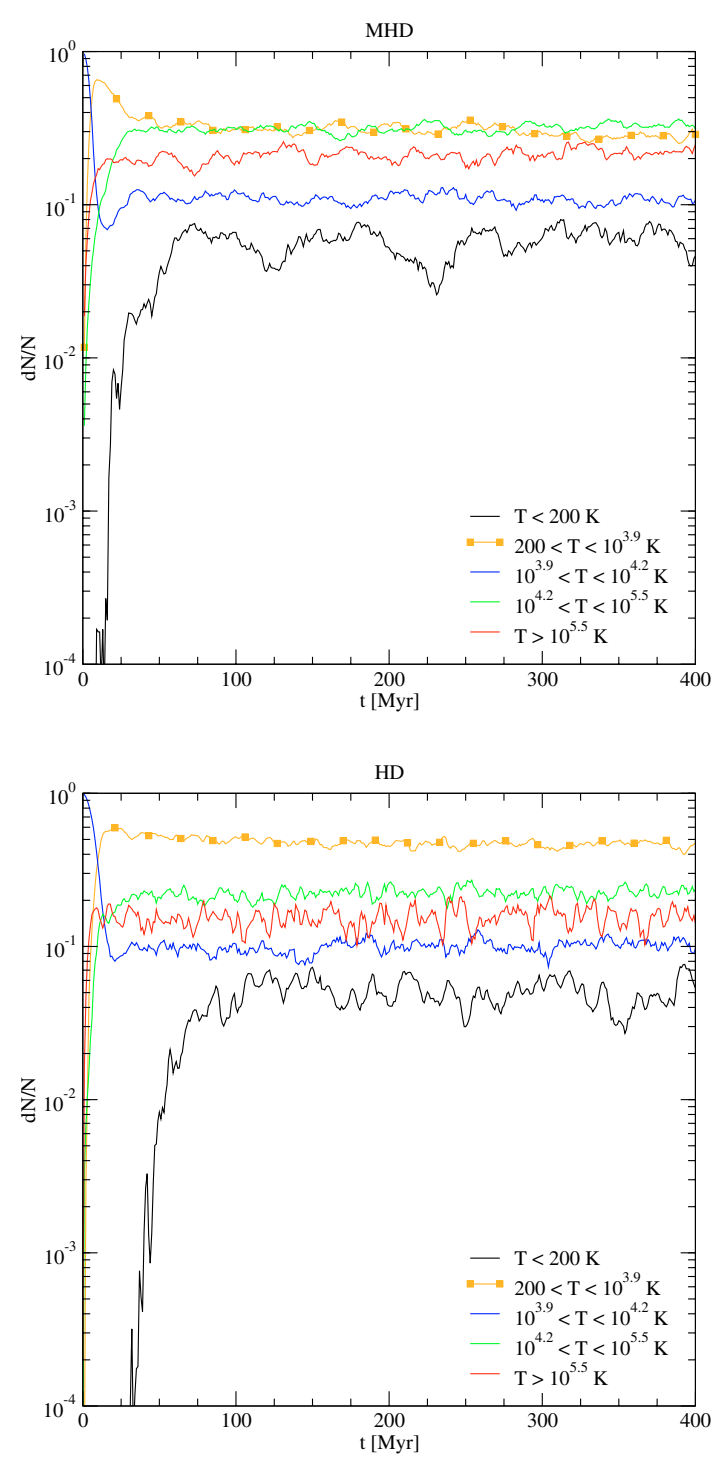

Fig. 8. Time history of the volume occupation fractions of the different temperature regimes for the MHD (top panel) and HD (bottom panel) runs.

\subsection{Volume and mass fractions}

During most of the history ( $t>100 \mathrm{Myr}$ ) of ISM simulation the occupation $\left(f_{\mathrm{v}}\right)$ and mass $\left(f_{\mathrm{M}}\right)$ fractions of the different thermal regimes (Figs. 8 and 9 - top and middle panels) have an almost constant distribution, varying around their mean values (cf. Table 1). The thermally stable regimes with $T \leq 200 \mathrm{~K}$ and $10^{3.9}<T \leq 10^{4.2} \mathrm{~K}$ have similar occupation fractions of $\sim 5 \%$ and $\sim 10 \%$, respectively, in both runs, while the hot gas has an increase from $\sim 17 \%$ in the HD run to $\sim 20 \%$ in the MHD case. By far most of the disk volume is occupied by gas in the thermally unstable regimes at $200<T \leq 10^{3.9}$ and $10^{4.2}<T \leq 10^{5.5} \mathrm{~K}$ with similar occupation fractions $\sim 30 \%$ in the MHD run, while in the HD run these regimes occupy $46 \%$ and $22 \%$, respectively, of the disk volume.

The major fraction of the disk mass ( $\sim 93 \%$ and $\sim 80 \%$ in the HD and MHD runs, respectively) is occupied by the gas with $T \leq 10^{3.9}$ and transferred between the cold and the thermally unstable regimes with the corresponding mass changes.
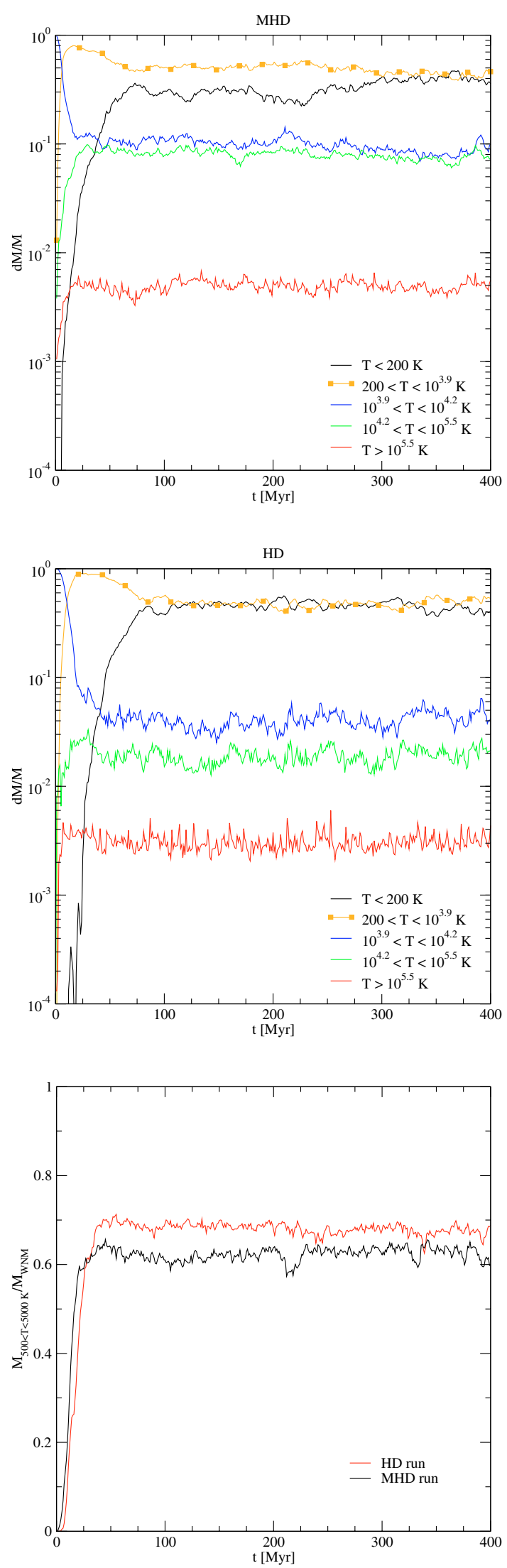

Fig. 9. Top and middle panels show the histories of the mass fractions of the different thermal regimes for the magnetized (top) and unmagnetized (middle) ISM. Bottom panel shows the history of the fraction of mass of the WNM gas having $500<T \leq 5000 \mathrm{~K}$ in the disk for the HD (red) and MHD (black) runs. 
Table 1. Summary of the average values of volume filling factors, mass fractions and root mean square velocities of the disk gas at the different thermal regimes for the HD and MHD runs.

\begin{tabular}{c|cc|cc|cc}
\hline \hline$T$ & \multicolumn{2}{|c|}{$\left\langle f_{\mathrm{v}}\right\rangle^{a}[\%]$} & \multicolumn{2}{|c|}{$\left\langle f_{\mathrm{M}}\right\rangle^{b}[\%]$} & \multicolumn{2}{|c}{$\left\langle v_{\mathrm{rms}}\right\rangle^{c}$} \\
\cline { 2 - 7 }$[\mathrm{K}]$ & HD & MHD & HD & MHD & HD & MHD \\
\hline$<200 \mathrm{~K}$ & 5 & 6 & 44.2 & 39.9 & 7 & 10 \\
$200-10^{3.9}$ & 46 & 29 & 49.0 & 43.7 & 15 & 15 \\
$10^{3.9}-10^{4.2}$ & 10 & 11 & 4.4 & 8.5 & 25 & 21 \\
$10^{4.2}-10^{5.5}$ & 22 & 33 & 2.0 & 7.4 & 39 & 28 \\
$>10^{5.5}$ & 17 & 21 & 0.3 & 0.5 & 70 & 55 \\
\hline
\end{tabular}

${ }^{a}$ Occupation fraction.

${ }^{b}$ Mass fraction.

${ }^{c}$ Root mean square velocity in units of $\mathrm{km} \mathrm{s}^{-1}$.

The cold $(T \leq 200 \mathrm{~K})$ regime during most of the simulation time encloses more mass than in the MHD run until $\sim 320 \mathrm{Myr}$ when both runs show a similar amount of cold mass $(\sim 40 \%)$, while the thermally unstable regime encloses the remaining mass. This results from the fact that in the MHD run the gas compression is smaller than in the HD case, because part of the shock energy goes into work against magnetic pressure. The remaining ISM mass is distributed between the other temperature regimes with the $10^{3.9}<T \leq 10^{4.2} \mathrm{~K}$ and $10^{4.2}<T \leq 10^{5.5} \mathrm{~K}$ regimes enclosing a total of $\sim 7 \%$ and $\sim 16 \%$ of mass in the HD and MHD runs, respectively, and the hot gas enclosing $<1 \%$ of the disk mass in both runs.

For most of the simulation time, in both runs, $60-70 \%$ of the warm neutral mass $(500<T \leq 8000 \mathrm{~K})$ is contained in the $500 \leq T \leq 5000 \mathrm{~K}$ temperature range (bottom panel of Fig. 9). This result in excellent agreement with the observations of Fitzpatrick \& Spitzer (1997) who found a value of $\approx 60 \%$ and Heiles \& Troland (2003) who determined a lower limit of $\approx 50 \%$ (see also Heiles 2001).

\subsection{Turbulent velocities}

In both the MHD and HD runs the root mean square (rms) velocity, $V_{\text {rms }}$, which is a measure of the disordered motion of the gas, increases with temperature as can be seen in Fig. 10, which shows the $V_{\text {rms }}$ for the different thermal regimes in the MHD (top panel) and HD (bottom panel) runs. The average rms velocity $\left(\left\langle V_{\mathrm{rms}}\right\rangle\right)$ in the last $100 \mathrm{Myr}$ of evolution of both media for the different thermal regimes is shown in Table 1. The figure and table show that there are large fluctuations in the $V_{\text {rms }}$ of the different thermal regimes in the HD run, which are reduced due to the presence of the magnetic field.

The rms velocities are in good agreement with those estimated from observations of cold/cool neutral (7-10 $\mathrm{km} \mathrm{s}^{-1}$; e.g., Kulkarni \& Fich 1985; Kulkarni \& Heiles 1987), warm neutral ( $14 \mathrm{~km} \mathrm{~s}^{-1}$; Kulkarni \& Fich 1985), warm ionized ( $\leq 30 \mathrm{~km} \mathrm{~s}^{-1}$; Reynolds 1985$)$ and hot $\left(\sim 33-78 \mathrm{~km} \mathrm{~s}^{-1}\right.$; e.g., Zsargó et al. 2003) gases. Again, the near constancy of the rms velocity with time indicates the presence of a dynamical equilibrium, with random motions, i.e. thermal and turbulent
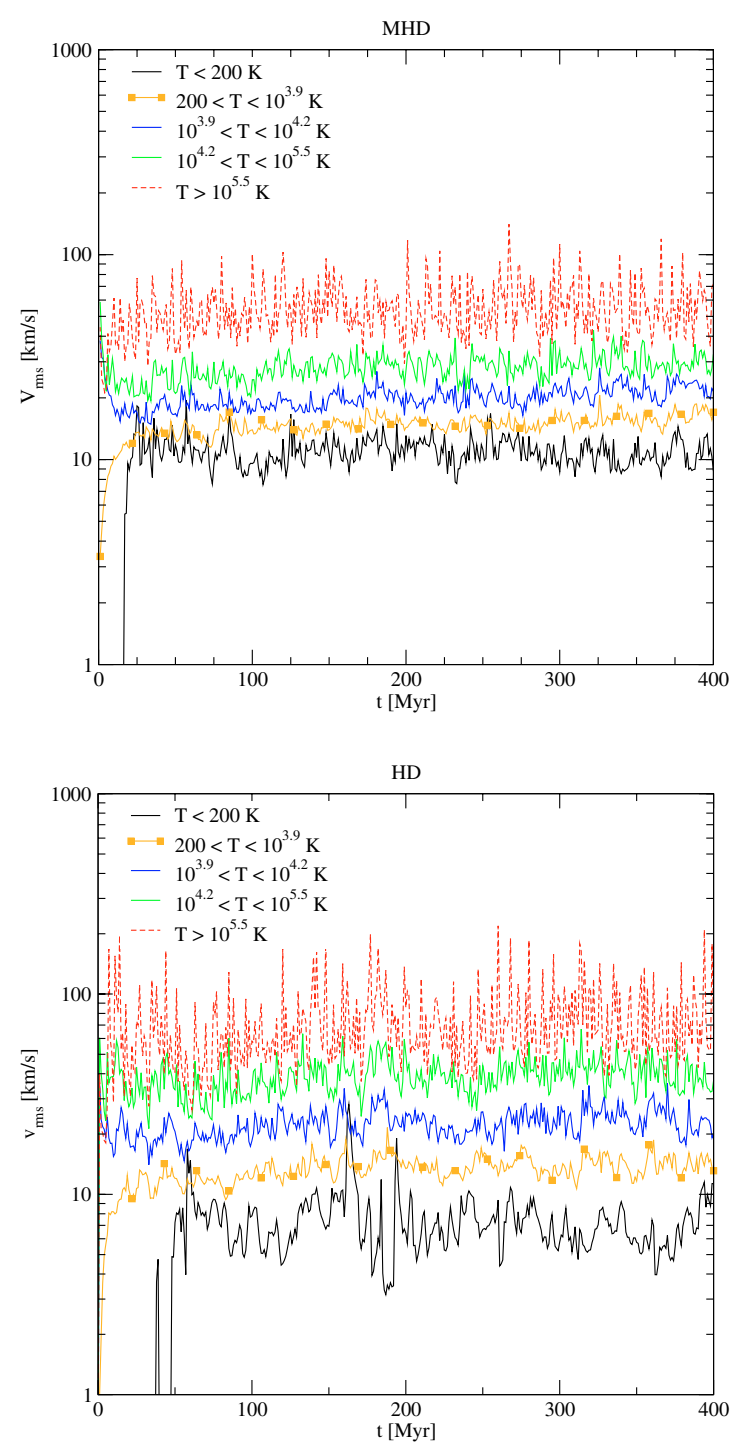

Fig. 10. History of the root mean square velocity of the different temperature regimes of the disk gas in the MHD (top panel) and HD (bottom panel) runs.

pressures adding to the total pressures, provided that the energy injection rate remains constant on a global scale.

\section{Discussion}

In the previous sections we compared high resolution threedimensional kpc-scale HD and MHD simulations of the ISM, driven by $\mathrm{SNe}$ at the Galactic rate, fully tracking the timedependent evolution of the large scale Galactic fountain (up to $10 \mathrm{kpc}$ on either side of the Galactic midplane) for a time sufficiently long (400 Myr) so that the memory of the initial conditions is completely erased, and a global dynamical equilibrium is established. These simulations show how important the establishment of the duty cycle is, and how it affects the global properties of the ISM, e.g., occupation fraction of the hot gas, just to name one. An essential feature of the hot gas is its escape into the halo establishing the fountain flow, thereby reducing the expansion volume in the underlying disk significantly. It is not possible to suppress such a flow even in the 
presence of an obstructing disk parallel magnetic field. All that is needed for break-out of the gas into the halo is a sufficient overpressure in the superbubbles with respect to the ambient medium. The effect of the magnetic field is just adding another pressure component. Its topology is almost irrelevant unless the galactic magnetic field is extremely high and/or the $\mathrm{SN}$ rate is very low. The field may inhibit the break-out of an individual remnant, but certainly not the high-pressure flow resulting from supernova explosions in concert within an OB association. Thus, the occupation fractions of the hot gas in the disk in the HD and MHD simulations are not too much different, although there is a slight increase in the MHD run as a result of the magnetic tension forces aiding to confine bubbles and Lorentz forces obstructing mixing with cooler gas. In Paper I we have shown that the volume filling factor of the hot regime strongly correlates with the supernova (and therefore star formation) rate. Although we have not carried out MHD simulations for higher supernova rates, it seems very plausible that this correlation will persist in the MHD case, since higher rates will produce more hot plasma which, as we have shown here, is not magnetically controlled.

We also emphasize the importance of carrying out threedimensional simulations in order to describe the evolution of a magnetized ISM adequately. The idea that a disk parallel magnetic field could suppress break-out and outflow into the halo was mainly based on 2D simulations carried out in the last 15 years, owing to computing power limitations. It is obvious, that in 2D-MHD, the flow perpendicular to the magnetic field lines (and hence to the galactic plane) is subject to opposing and ever increasing magnetic tension and pressure forces. In 3D however, field lines can be pushed aside and holes and channels can be punched into the decreasing gas and field with $z$-height, allowing pressurized flow to circumvent increasing tension and pressure forces in z-direction. It is exactly this behaviour that we see in our simulations. This is less surprising if we think of similar problems of terrestrial plasma confinement, although here plasma instabilities prevail.

The lack of correlation between the field strength and density found in the present simulations is supported observationally from measurements of the magnetic field strength in the cold neutral medium (Troland \& Heiles 2001). A similar result is shown in the simulation described in Kim et al. (2001), although these authors claim that the magnetic field scales as $\rho^{0.4}$ at densities $n>1 \mathrm{~cm}^{-3}$. Their Fig. 2 shows an almost order of magnitude variation of the field with the density for the coolest gas. However, the distribution of the scatter points is somewhat different than that seen in Fig. 4 of the present paper. This discrepancy can be explained by the $(200 \mathrm{pc})^{3}$ box that Kim et al. have used, centered in the Galactic midplane having no density stratification and with periodic boundary conditions in all the box faces, driven by $\mathrm{SNe}$ at a rate of 12 times the Galactic value and using an uniform field strength of $5.8 \mu \mathrm{G}$ orientated along the $x$-direction; thus they were unable to describe the disk-halodisk circulation and did not allow for a global dynamical equilibrium to be established (see also Mac Low et al. 2004).

The distribution of the disk mass in the warm neutral medium (WNM), in particular in the WNM fraction within the classical thermally unstable regime seen in the simulations is strongly supported by interferometric (Kalberla et al. 1985). Moreover optical/UV absorption-line measurements (Spitzer \& Fitzpatrick 1995; Fitzpatrick \& Spitzer 1997) indicate that a large fraction $(\sim 63 \%)$ of the warm neutral medium (WNM) is in the unstable range $500<T<5000 \mathrm{~K}$, whereas $21 \mathrm{~cm}$ line observations (Heiles 2001; Heiles \& Troland 2003) provide a lower limit of $48 \%$ for the WNM gas in this unstable regime. Direct numerical simulations of the nonlinear development of the thermal instability under ISM conditions with radiative cooling and background heating discussed in Gazol et al. (2001) and Kritsuk \& Norman (2002) show that about $60 \%$ of the system mass is in the thermally unstable regime. However, it is unclear from their simulations what is the time evolution of this mass fraction and what is explicitly the origin of the unstable gas, although these authors suggest that ensuing turbulence is capable of replenishing gas in the thermally unstable regime by constantly stirring up the ISM. We have carried out detailed numerical studies of the stability of the ISM gas phases (Avillez \& Breitschwerdt 2005), and could verify the hypothesis that SN driven turbulence is capable of replenishing fast cooling gas in classically unstable regimes.

Nonetheless, it is interesting to ask why such a large amount of gas can exist in a thermally unstable regime. Obviously the simple Field (1965) criterion, according to which instability is expected to set in, viz. $\left(\frac{\partial \mathcal{L}}{\partial T}\right)_{P}<0$, where $\mathcal{L}$ is the heat loss function per unit mass, and $T$ and $P$ are the temperature and thermal pressure of the fluid element, respectively, is not adequate. It is the turbulence that can have a stabilizing effect thereby inhibiting local condensation modes. The situation is reminiscent to the existence of the solar chromosphere, which consists of gas at around $10^{5} \mathrm{~K}$, again in the thermally unstable regime. The reason is because heat conduction can prevent thermal runaway on small scales. In other words, diffusion processes have a stabilizing effect. In our case it is turbulent diffusion that replaces the rôle of conduction, again, most efficient for large wavenumbers. The turbulent viscosity $v_{\text {turb }} \sim \operatorname{Re} v_{\text {mol }}$ can be orders of magnitude above the molecular viscosity, with Re being the Reynolds number of the flow. What happens physically then, is that with increasing eddy wavenumber $k=2 \pi / \lambda$, the eddy crossing time $\tau_{\text {eddy }} \sim \lambda / \Delta u$ (with $\Delta u$ being the turbulent velocity fluctuation amplitude) becomes shorter than the cooling time $\tau_{\text {cool }} \sim 3 k_{\mathrm{B}} T /(n \Lambda(T))$, where $\Lambda(T)$ is the interstellar cooling function. Although not really applicable here, it is instructive to see that in case of incompressible turbulence following a Kolmogoroff scaling law, where the energy dissipation rate is given by $\varepsilon \sim \rho \Delta u^{3} / \lambda$, we obtain a lower cut-off in wavelength, where thermal instability becomes inhibited, if

$$
\begin{aligned}
\lambda & <\left(\frac{3 k_{\mathrm{B}} \bar{m}}{\Lambda_{0}}\right)^{3 / 2} \varepsilon^{1 / 2} \frac{T^{3 / 4}}{\rho^{2}} \\
& \approx 1.42 \times 10^{19} \mathrm{~cm},
\end{aligned}
$$

taking $\varepsilon \sim 10^{-26} \mathrm{erg} \mathrm{cm}^{-3} \mathrm{~s}^{-1}$ for SN energy injection; a simple cooling law for the warm neutral medium of $\Lambda(T)=\Lambda_{0} T^{1 / 2}$ has been adopted, with $\Lambda_{0} \approx 1.9 \times 10^{-27} \mathrm{erg} \mathrm{cm}^{3} \mathrm{~s}^{-1} \mathrm{~K}^{-1 / 2}$ (taken from the cooling curve of Dalgarno \& McCray 1972) for a WNM of a density of $n=0.3 \mathrm{~cm}^{-3}$, a temperature of 
$T=1000 \mathrm{~K}$, and a low degree of ionization $x \approx 0.01$. Therefore rough numerical estimates are typically of the order of parsecs, consistent with our numerical resolution. In fact, the critical wavelength $\lambda$ varies with temperature, degree of ionization and hence cooling; for the WNM we find quite a large range of values from $10^{17}-10^{20} \mathrm{~cm}$, according to Eq. (21). We will investigate the case of compressible, superalfvenic and supersonic turbulence in more detail in a forthcoming paper.

A concern with the present simulations is the numerical resolution, which is always a limitation, to which HD/MHD simulations are subject. The crucial questions are (i) how do the results change as the resolution is increased; and (ii) how is the amount of cold gas affected by a resolution larger than the Field length, $\lambda_{\mathrm{F}}=0.1 \mathrm{pc}$ in the WNM and $0.001 \mathrm{pc}$ in the CNM (see e.g., Audit \& Hennebelle 2005), far below the limit one can handle in global simulations. Note that both Koyama \& Initsuka (2002) and Audit \& Hennebelle (without magnetic fields) used 2D boxes of $0.3 \mathrm{pc}$ and $20 \mathrm{pc}$ in length, three and two orders of magnitude smaller than our box size and cell resolutions of the order of 0.01 parsec. The first of these questions has been discussed at length for the HD run in Paper I, where it has been shown that the large scale structure and global properties of the ISM of the different thermal regimes remain essentially unchanged, once a minimum resolution of $1.25 \mathrm{pc}$ is achieved. With regard to (ii) the calculation of the cooling length depends also on heat conduction, which may be suppressed in stochastic magnetic fields as the ones seen in the present simulations (for a detailed discussion see Paper I; see also Malyshkin \& Kulsrud 2001). However, as shown above in a dynamical ISM the Field criterion can be superseded, as the cooling fluid element may be overturned by turbulent fluctuations in the velocity and density field and therefore, some of the gas that is heated up or cooling down to an unstable regime may not participate in a transition to a stable phase at lower temperature.

The present simulations still neglect an important component of the ISM, i.e. high energy particles, which are known to be in rough energy equipartition locally with the magnetic field, the thermal and the turbulent gas in the ISM. The presence of CRs and magnetic fields in galactic halos is well known and documented by many observations of synchrotron radiation generated by the relativistic electron component. There is increasing evidence that the fraction of these cosmic rays that dominates their total energy is of Galactic origin and can be generated in supernova remnants via the so-called diffusive shock acceleration mechanism to energies up to $10^{15} \mathrm{eV}$ (for original papers see Krymski et al. 1977; Axford et al. 1977; Bell 1978; Blandford \& Ostriker 1978, for a review Drury 1983, for more recent calculations see Berezhko 1996). It is also common wisdom that the propagation of these particles generates MHD waves due to the so-called streaming instability (e.g. Kulsrud \& Pearce 1969) and thereby enhances the turbulence in the ISM. In addition, self-excited MHD waves will lead to a dynamical coupling between the cosmic rays and the outflowing fountain gas, which will enable part of it to leave the galaxy as a galactic wind (Breitschwerdt et al. 1991, 1993; Dorfi \& Breitschwerdt 2005). Furthermore, as the cosmic rays act as a weightless fluid, not subject to radiative cooling, they can bulge out magnetic field lines through buoyancy forces. Such an inflation of the field will inevitably lead to a Parker type instability, and once it becomes nonlinear, it will break up the field into a substantial component parallel to the flow (Kamaya et al. 1996), thus facilitating gas outflow into the halo. It has been suggested that eventually reconnection with an undisturbed halo field component (Tanuma et al. 2003) and/or due to twisted approaching flux tubes driven by Coriolis forces (Hanasz et al. 2002), will dissipate some of the field energy and change the field topology. This may even provide the necessary ingredients for a Galactic $\alpha \omega$-dynamo to operate, generating a large scale poloidal field as was originally proposed by Parker (1992). It is still a matter of debate, if Galactic halo conditions are suitable for anomalous resistivity to occur in current sheets with a minute thickness of the ion Larmor radius in order to promote fast reconnection at about $10 \%$ of the Alfvén speed. In any case will such a scenario alleviate the transport of hot plasma into the halo. The effect of cosmic rays on large scale ISM evolution will also be the subject of a forthcoming paper.

\section{Summary and final remarks}

In the present paper we investigate the rôle of magnetic field and matter circulation between the disk and halo and its effect on the dynamical evolution of the disk gas. These results are compared to HD simulations of the same resolution that we have carried out as well. The main results of this paper can be summarized as follows:

- The simulations show the presence of a wiggly thin disk of cold gas (both in density as well in magnetic field) overlayed by a frothy thick disk composed of neutral and ionized hydrogen having different scale heights.

- The thin magnetized disk is overlayed by Parker-like loops (produced without cosmic rays), magnetic islands, clouds wrapped in field lines moving downwards and cold gas descending along the Parker loops, which have risen to several kpc above the plane within $130 \mathrm{Myr}$.

- The highest density gas tends to be confined into shock compressed layers that form in regions where several large scale streams of convergent flow (driven by $\mathrm{SNe}$ ) occur.

- The compressed regions, which have on average lifetimes of a few free-fall times (about 10-15 Myr), are filamentary in structure, tend to be aligned with the local field and are associated with the highest field strengths (in the MHD run), while in the HD run there is no preferable orientation of the filaments. The formation time of these high density structures depends on how much mass is carried by the convergent flows, how strong the compression and what the rate of cooling of the regions under pressure are (see also Ballesteros-Paredes 2004).

- The magnetic field has a high variability (which decreases towards higher gas densities) and it is largely uncorrelated with the density. The field is driven by inertial motions (which is consistent with the dominance of the ram pressure), rather than it being the agent determining the gas motions. In the latter case the field would not be strongly 
distorted, and it would direct the motions predominantly along the field lines.

- $T \leq 200 \mathrm{~K}$ gas has $P_{\mathrm{B}}>P_{\text {ram }} \gg P_{\text {th }}$, demonstrating that magnetically dominated regions do exist. For $200<T \leq$ $10^{5.5} \mathrm{~K}$ ram pressure determines the dynamics of the flow, and therefore, the magnetic pressure does not act as a significant restoring force. Near supernovae thermal and ram pressures determine the dynamics of the flow. The hot gas in contrast is controlled by the thermal pressure, since magnetic field lines are swept towards the dense compressed walls.

- The existence of ram pressure dominated flows leads inevitably to shear flows, thus driving strong superalfvenic and supersonic turbulence in the ISM.

- The volume filling factors of the different ISM temperature regimes depend sensitively on the existence of a duty cycle between the disk and halo acting as a pressure release valve for the hot $\left(T>10^{5.5} \mathrm{~K}\right)$ gas in the disk. The mean occupation fraction of the hot phase in both $H D$ and MHD runs is $\sim 17-21 \%$. The $T \leq 200 \mathrm{~K}$ and $10^{3.9}<T \leq 10^{4.2} \mathrm{~K}$ regimes occupy $5-6 \%$ and $10-11 \%$ of the disk volume, respectively, while the thermally unstable regimes $\left(200<T \leq 10^{3.9}\right.$ and $\left.10^{4.2}<T \leq 10^{5.5} \mathrm{~K}\right)$ fill in total up to $70 \%$ of the disk volume in both runs.

- Most of the disk mass is found in the $T \leq 10^{3.9} \mathrm{~K}$ gas, with the cold $(T \leq 200 \mathrm{~K})$ and thermally unstable gases $\left(200<T \leq 10^{3.9} \mathrm{~K}\right.$ ) harbouring on average 83 and $93 \%$ of the disk mass in the MHD and HD runs. About $64-67 \%$ of the WNM gas has temperatures in the range 500-5000 K in both runs.

- With the magnetic field present and initially orientated parallel to the disk varying as $\rho^{1 / 2}$, transport into the halo is inhibited but not prevented. On larger scales magnetic tension forces are weaker than on the smallest scales and therefore vertical expansion still takes place efficiently and the occupation fraction of the hot gas becomes comparable to the values observed in the hydrodynamical simulations. Thus, hot gas is fed into the Galactic fountain at almost a similar rate than without the field.

In the dynamical picture of the ISM emerging from our 3D high-resolution simulations, turbulence generated by shear flows of expanding bubbles seems to be a key element of structure formation in both HD and MHD runs. Starting from here and following a bottom-up scheme we feel encouraged to study in the future other physical processes and ingredients in more detail, such as non-equilibrium cooling, self-gravity, heat conduction and cosmic rays, respectively, to name the most important ones.

Acknowledgements. M.A. and D.B. are partially supported by the ESO/FCT (Portuguese Science foundation) grant PESO/P/PRO/40149/2000. D.B. thanks G. Hensler for useful discussions on the subject. M.A. benifited from discussions with E. Vazquez-Semadeni and M.-M. Mac Low.

\section{References}

Audit, E., \& Hennebelle, P. 2005, A\&A, in press [arXiv: astro-ph/0410062]
Avillez, M. A. 1998, The Evolution of Galactic Fountains, Ph.D. Thesis, University of Évora, Portugal

Avillez, M. A. 2000, MNRAS, 315, 479

Avillez, M. A., \& Breitschwerdt, D. 2004, A\&A, 425, 899 (Paper I)

Avillez, M. A., \& Breitschwerdt, D. 2005, ApJ, in preparation

Axford, W. I., Leer, E., \& Skadron, G. 1977, in Proc. 15th Int. Cosmic Ray Conf. (Plodiv) 11, 132

Ballesteros-Paredes, J. 2004, Ap\&SS, 289, 243

Balsara, D. S. 2001, J. Comput. Phys., 174, 614

Bell, A. R. 1978, MNRAS, 182, 147

Beck, R. 2004, Ap\&SS, 289, 293

Berezhko, E. G. 1996, APh, 5, 367

Blandford, R. D., \& Ostriker, J. P. 1978, ApJ, 221, 29

Bregman, J. N., \& Pildis, R. A. 1994, ApJ, 420, 570

Breitschwerdt, D., McKenzie, J. F., \& Völk, H. J. 1991, A\&A, 245, 79

Breitschwerdt, D., McKenzie, J. F., \& Völk, H. J. 1993, A\&A, 269, 54

Breitschwerdt, D., \& Schmutzler, T. 1994, Nature, 371, 774

Brinks, E., \& Bajaja, E. 1986, A\&A, 169, 14

Chandrasekhar, S., \& Fermi, E. 1953, ApJ, 118, 113

Dai, W., \& Woodward, P. R. 1994, J. Comput. Phys., 115, 485

Dai, W., \& Woodward, P. R. 1998, J. Comput. Phys., 142, 331

Dettmar, R.-J. 1992, Fund. Cosm. Phys., 15, 143

Dorfi, E. A., \& Breitschwerdt, D. 2005, A\&A, in preparation

Drury, L. Oc. 1983, Rep. Prog. Phys., 46, 973

Ferrière, K. M., Mac Low, M.-M., \& Zweibel, E. G. 1991, ApJ, 375, 239

Field, G. B. 1965, ApJ, 142, 531

Field, G. B., Goldsmith, D. W., \& Habing, H. J. 1969, ApJ, 155, L149

Fitzpatrick, E. L., \& Spitzer, L., Jr. 1997, ApJ, 475, 623

Gazol, A., Vázquez-Semadeni, E., Sanchez-Salcedo, J., \& Scalo, J. 2001, ApJ, 557, 121

Guiliani, J. L., Jr. 1982, ApJ, 256, 624

Hanasz, M., Otmianowska-Mazur, K., \& Lesch, H. 2002, A\&A, 386, 347

Hartmann, J. 1904, ApJ, 19, 268

Heiles, C. 2001, ApJ, 551, L105

Heiles, C., \& Troland, T. H. 2003, ApJ, 586, 1067

Kahn, F. D. 1981, in Investigating the Universe, ed. F. D. Kahn (Dordrecht: Reidel), 1

Kamaya, H., Mineshige, S., Shibata, K., \& Matsumoto, R. 1996, ApJ, 458, L25

Kalberla, P. M. W., Schwarz, U. J., \& Goss, W. M. 1985, A\&A, 144, 27

Kim, J., Balsara, D., \& Mac Low, M.-M. 2001, JKAS, 34, S333

Koo, B.-C., \& McKee, C. F. 1992, ApJ, 388, 93

Korpi, M. J., Brandenburg, A., Shukurov, A., Tuominen, I., \& Nordlund, A. 1999, ApJ, 514, L99

Koyama, H., \& Inutsuka, S. 2002, 564, L97

Krymsky, G. F. 1977, Dokl. Nauk. SSR, 234, 1306 (Engl. Trans. Sov. Phys. Dokl., 23, 327)

Kritsuk, A., \& Norman, M. L. 2002, ApJ, 569, L127

Kulkarni, S. R., \& Fich, M. 1985, ApJ, 289, 792

Kulkarni, S. R., \& Heiles, C. 1987, in Interstellar Processes, ed. D. J. Hollenbach, \& H. A. Thronson (Dordrecht: Reidel), 87

Kulsrud, R. M., Bernstein, I. B., \& Kruskal, M. 1965, ApJ, 142, 491

Kulsrud, R. M., \& Pearce, W. D. 1969, ApJ, 156, 445

Lockman, F. J. 1984, ApJ, 283, 90

Mac Low, M.-M., Balsara, D., Kim, J., \& Avillez, M. A. 2004, ApJ, in revision [arXiv: astro-ph/0410734]

Malyshkin, L., \& Kulsrud, R. 2001, ApJ, 549, 402

Massey, P., Johnson, K. E., \& Degioia-Eastwood, K. 1995, ApJ, 454, ApJ, 454, 151

McCray, R., \& Kafatos, M. 1987, ApJ, 317, 190 
McKee, C. F., \& Ostriker, J. P. 1977, ApJ, 218, 148

Mineshige, S., Shibata, K., \& Shapiro, P. R. 1993, ApJ, 409, 663

Norman, C. A., \& Ikeuchi, S. 1989, ApJ, 345, 372

Parker, E. N. 1992, ApJ, 401, 137

Passot, T., \& Vázquez-Semadeni, E. 2003, A\&A, 398, 845

Reynolds, R. J. 1985, ApJ, 294, 256

Spitzer, L., Jr. 1956, ApJ, 124, 20

Spitzer, L., Jr., \& Fitzpatrick, E. L. 1995, ApJ, 445, 196

Stothers, R. 1972, ApJ, 175, 431

Summers, D. 1975, A\&A, 45, 151

Tanuma, S., Yokoyama, T., Kudoh, T., \& Shibata, K. 2003, ApJ, 582, 215

Tomisaka, K. 1990, ApJ, 361, L5

Tomisaka, K. 1998, MNRAS, 298, 797
Troland, T. H., \& Heiles, C. 2001, BAAS, 33, 918

Trümper, J. 1983, Adv. Sp. Res., 2(4), 241

Vogler, A., \& Pietsch, W. 1996, A\&A, 311, 35

Wang, Q. D., Walterbos, R. A. M., Steakley, M. F., Norman, C. A., \& Braun, R. 1995, ApJ, 439, 176

Wang, Q. D., Immler, S., Walterbos, R., Lauroesch, J. T., \& Breitschwerdt, D. 2001, ApJ, 555, L99

Weaver, R., McCray, R., Castor, J., Shapiro, P., \& Moore, R. 1977, ApJ, 218, 377

Wolfire, M. G., McKee, C. F., Hollenbach, D., Tielens, A. G. G. M., \& Bakes, E. L. O. 1995, ApJ, 443, 152

Zsargó, J., Sembach, K. R., Howk, J. C., \& Savage, B. D. 2003, ApJ, 586, 1019 